\title{
Imaginary quadratic fields with small odd class number
}

\author{
by \\ Steven Arno, M. L. Robinson and \\ Ferrell S. Wheeler (Bowie, Md.)
}

1. Introduction. Let $-d$ be the discriminant of an imaginary quadratic field with class number $h(-d)$. As is well known, Gauss [9] conjectured that $h(-d)$ tends to infinity with $d$. Hence, for fixed $m$, it was natural to ask for a complete list of negative fundamental discriminants $-d$ such that $h(-d)=m$. This problem is usually referred to as Gauss' class number problem or Gauss' class number $m$ problem.

In 1934, Heilbronn [14] succeeded in proving Gauss' conjecture, thereby placing the class number problem on firm ground. The following year, Siegel [20] showed that for any $\varepsilon>0$ there exists a constant $c_{\varepsilon}>0$ such that $h(-d)>c_{\varepsilon} d^{1 / 2-\varepsilon}$ as $d \rightarrow \infty$. Unfortunately, neither result was effective, and no further progress was made until the 1950's when Heegner [13] offered a solution for the class number 1 problem based on new ideas from the theory of modular functions. It is interesting to recall that Heegner's proof was generally discounted until the "gaps" in his argument were explained many years later (see [5], [8], [21], [24]). In the interim period, however, the first accepted proof of the class number 1 problem was given by Stark [23] in 1966. Shortly thereafter, Baker $[2,4]$ found another proof based on the theory of transcendental numbers. In 1971, Baker [3, 4] and Stark [26-28] independently resolved the class number 2 problem as well. However, there seemed to be little hope of generalizing these methods to solve higher class number problems.

In 1976, Goldfeld [10] presented a deep and entirely unexpected result which provided the framework for a general attack on the class number problem. He showed that if there exists a Weil curve whose associated $L$-function has a zero of at least the third order at $s=1$, then for any $\varepsilon>0$

1991 Mathematics Subject Classification: Primary 11R; Secondary 11Y, 11J.

Key words and phrases: binary quadratic forms, imaginary quadratic fields, class numbers, discriminants. 
there exists an effectively computable constant $c_{\varepsilon}$ such that

$$
h(-d)>c_{\varepsilon}(\log d)^{1-\varepsilon} .
$$

Of course, the utility of Goldfeld's result depended on finding an appropriate elliptic curve. And though one would expect to find a Weil curve with a high order zero at $s=1$ based on the celebrated conjecture of Birch and Swinnerton-Dyer [5], technical difficulties kept things on hold for several years. Finally, in 1983 Gross and Zagier [11] were able to show that certain curves must have a zero of at least the third order at $s=1$, thereby completing the attack of Goldfeld.

Goldfeld's proof was later simplified by Oesterlé [19], who provided, among other things, explicit constants for Goldfeld's theorem. As a result, Oesterlé was able to complete the class number 3 problem as well. The class number 4 problem was solved by the first author [1] who combined new techniques with the well-known methods of Stark [22], MontgomeryWeinberger [18], and Oesterlé [19]. In hindsight, [1] contains a prototype for the partitioning of minima (of reduced quadratic forms of discriminant $-d$ ), which plays a crucial role in this paper.

The central concern of this paper is the class number $m$ problem for small, odd $m$. The aforementioned partitioning of minima enables us to significantly improve on earlier estimates. Our results are summarized in the following theorem.

Theorem 1. For each odd integer $m$ satisfying $5 \leq m \leq 23$, the class number $m$ problem is solved. For each such $m$, a complete list of negative fundamental discriminants $-d$ for which $h(-d)=m$ can be found in Appendix A.

Let $-d$ be the discriminant of an imaginary quadratic field with class number $h(-d)$. In Table 1 we present the number of fields satisfying $h(-d)=m$ and the largest such $d$ for each odd $m$ satisfying $1 \leq m \leq 23$.

Table 1. Upper bound on $d$ satisfying $h(-d)=m$

\begin{tabular}{rrr}
\hline$m$ & \# of $d$ & max. $d$ \\
\hline 1 & 9 & 163 \\
3 & 16 & 907 \\
5 & 25 & 2683 \\
7 & 31 & 5923 \\
9 & 34 & 10627 \\
11 & 41 & 15667 \\
13 & 37 & 20563 \\
15 & 68 & 34483 \\
17 & 45 & 37123 \\
19 & 47 & 38707 \\
21 & 85 & 61483 \\
23 & 68 & 90787 \\
\hline
\end{tabular}


The paper is organized as follows. In $\S 2$ we use Oesterlé's [19] explicit constants to produce a $d_{3}(m)$ such that if $d \geq d_{3}(m)$, then $h(-d) \neq m$. In $\S 3$ we provide the theoretical justification for the partitioning of minima mentioned above. In $\S 4$ we prove several technical lemmas concerning an auxiliary function which are needed in $\S 5$.

We divide $\S 5$ into three subsections, each of which rules out a certain range of fundamental discriminants. In $\S 5.1$, following the approach of Montgomery-Weinberger [18], we produce a $d_{2}(m)$ such that if $d_{2}(m) \leq$ $d \leq d_{3}(m)$, then $h(-d) \neq m$. At the end of $\S 5.1$ we note that the methods of $\S 5.1$, even when pushed to their limits, do not reduce the range of admissible discriminants $d$ to the point where a computationally intensive sieve (like the one introduced in $\S 6$ ) can be used to complete the class number $m$ problem when $m>7$. This shows that some new argument is necessary. In $\S 5.2$ we exploit the partitioning of minima introduced in $\S 3$ to produce a $d_{1}(m)$ such that if $d_{1}(m) \leq d \leq d_{2}(m)$, then $h(-d) \neq m$. When $m$ is odd and $5 \leq m \leq 13, d_{1}(m)$ is small enough to allow the class number $m$ problem to be completed with the computationally intensive sieve of $\S 6$. In $\S 5.3$ we use a more sophisticated version of the arguments in $\S 5.2$ to produce a $d_{0}(m)$ such that if $d_{0}(m) \leq d \leq d_{1}(m)$, then $h(-d) \neq m$. When $m$ is odd and $15 \leq m \leq 23, d_{0}(m)$ is small enough to allow the class number $m$ problem to be completed with the computationally intensive sieve of $\S 6$. We combine the partitioning of minima with the aforementioned sieve to complete the proof of Theorem 1 in $\S 6$.

Notation. Let $\mathbb{Z}, \mathbb{Z}^{+}, \mathbb{Q}, \mathbb{R}$ and $\mathbb{C}$ denote the ring of integers, the set of positive integers, the field of rational numbers, the field of real numbers, and the field of complex numbers, respectively. Let $p$ and $q$ denote primes in $\mathbb{Z}^{+}$. The Kronecker symbol is denoted by either $\left(\frac{m}{n}\right)$ or $(m \mid n)$, depending on which is more convenient. As is customary, we let $\omega(n)$ denote the number of distinct prime divisors of $n$ and $d(n)$ the total number of positive divisors of $n$. Finally, let $e(x)=e^{2 \pi i x}$.

The number $-d$ denotes a negative fundamental discriminant or, equivalently, the discriminant of an imaginary quadratic number field. In other words, we have either $-d \equiv 1(\bmod 4)$ and $d$ is square-free, or $4 \mid d$ and $-d / 4 \equiv 2$ or $3(\bmod 4)$ and $d / 4$ is square-free. $\chi_{1}$ denotes the real primitive character with $\chi_{1}(n)=(-d \mid n)$.

A binary quadratic form

$$
Q(x, y)=a x^{2}+b x y+c y^{2}
$$

of discriminant $-d=b^{2}-4 a c$ is reduced if it satisfies either

$$
-a<b \leq a<c \quad \text { or } \quad 0 \leq b \leq a=c \quad(a, b, c \in \mathbb{Z}) .
$$


Note that this implies

$$
a \leq(d / 3)^{1 / 2}
$$

Let

$$
Q_{d}=\left\{Q(x, y)=a x^{2}+b x y+c y^{2}: b^{2}-4 a c=-d, Q \text { is reduced }\right\}
$$

denote the finite set of reduced binary quadratic forms with discriminant $-d$. Note $h(-d)=\left|Q_{d}\right|$. The notation $\sum_{Q_{d}}$ denotes a sum over all $Q \in Q_{d}$ along with the associated coefficients $a, b$, and $c$.

The modified Bessel function of the second kind of order zero is given by

$$
K_{0}(z)=\int_{1}^{\infty} e^{-(z / 2)\left(t+t^{-1}\right)} \frac{d t}{t} \quad(\Re z>0) .
$$

2. The high range. As mentioned in $§ 1$, Oesterlé [19] provided explicit constants for the $c_{\varepsilon}$ in Goldfeld's inequality (1.1). Indeed, if $-d$ is a negative fundamental discriminant with class number $h(-d)$ and

$$
\vartheta(d)=\prod_{p \mid d}^{*}\left(1-\frac{\lfloor 2 \sqrt{p}\rfloor}{p+1}\right),
$$

where the product is taken over all prime divisors $p$ of $d$ with the exception of the largest prime divisor, then

$$
\vartheta(d) \log d \leq C h(-d)
$$

where $C=55$ if $(d, 5077)=1$ and $C=7000$ otherwise.

In order to evaluate $\vartheta(d)$ when $h(-d)$ is odd, recall that the Fundamental Theorem of Genera due to Gauss [9] implies that

$$
2^{\omega(d)-1} \mid h(-d) \text {. }
$$

If $h(-d)$ is odd, then the preceding result implies that $d$ has only one prime divisor. Since $-d$ is a discriminant, we see that $-d$ is either $-4,-8$, or $-p$ for some odd prime $p \equiv 3(\bmod 4)$. It follows that when $h(-d)$ is odd, $\vartheta(d)=1$. Since $h(-4)=h(-8)=1$, we have

$$
h(-d) \text { is odd and } h(-d)>1 \Rightarrow d \text { is prime and } d \equiv 3(\bmod 4) \text {. }
$$

Hence, when $h(-d)$ is odd and $h(-d)>1,(2.1)$ implies that $(d, 5077)=1$ (for 5077 is a prime equal to 1 modulo 4), and we have

$$
\log d \leq 55 h(-d)
$$

Thus, for $m \in\{5,7, \ldots, 23\}$, we have $h(-d)>m$ if $d \geq d_{3}(m)$ where $d_{3}(m)$ is given in Table 2 . 
Table 2. $h(-d) \neq m$ for $d \geq d_{3}(m)$

\begin{tabular}{rc}
\hline$m$ & $d_{3}(m)$ \\
\hline 5 & $10^{120}$ \\
7 & $10^{168}$ \\
9 & $10^{215}$ \\
11 & $10^{263}$ \\
13 & $10^{311}$ \\
15 & $10^{359}$ \\
17 & $10^{407}$ \\
19 & $10^{454}$ \\
21 & $10^{502}$ \\
23 & $10^{550}$ \\
\hline
\end{tabular}

Note that when $h(-d)$ is even, $d$ may be composite. This allows the possibility that a minimum $a$ of a reduced form could satisfy $(a, d)>1$. This introduces many technical difficulties, the least of which is a smaller value of $\vartheta(d)$. For these reasons, we confine our attention to the case where $h(-d)$ is odd.

3. Minima results. The coefficient $a$ is referred to as the minimum of the form $a x^{2}+b x y+c y^{2} \in Q_{d}$, while the multiset of minima for a given negative fundamental discriminant $-d$ is denoted by

$$
M_{d}=\left\{a: a x^{2}+b x y+c y^{2} \in Q_{d}\right\} .
$$

From (1.2) it is easy to see that 1 occurs in $M_{d}$ precisely once. We henceforth refer to $1 \in M_{d}$ as the principal minimum since 1 is the minimum of the principal form (i.e., either $x^{2}+(d / 4) y^{2}$ or $x^{2}+x y+((d+1) / 4) y^{2}$ depending on the parity of $d$ ). Furthermore, we note that all elements of $M_{d}$ are positive by (1.2).

Lemma 1. If $a \in M_{d}$, then any positive divisor of $a$ is also in $M_{d}$.

Proof. Let $a_{1}>0$ be a divisor of $a$. If $a_{1}=a$ there is nothing to prove. Hence, we may assume $a_{1} \leq a / 2$. It follows from (1.3) that

$$
a_{1} \leq(d / 12)^{1 / 2} .
$$

Let $a x^{2}+b x y+c y^{2} \in Q_{d}$ so that $b^{2}-4 a c=-d$. It follows at once that the quadratic congruence $z^{2} \equiv-d\left(\bmod 4 a_{1}\right)$ is solvable. Hence, there exists a $b_{1}$ such that $b_{1}^{2} \equiv-d\left(\bmod 4 a_{1}\right)$, with $-2 a_{1}<b_{1} \leq 2 a_{1}$. Further, this range can be sharpened to ensure that $-a_{1}<b_{1} \leq a_{1}$ by replacing $b_{1}$ with $b_{1}-2 a_{1}$ if $a_{1}<b_{1} \leq 2 a_{1}$ and $b_{1}$ with $b_{1}+2 a_{1}$ if $-2 a_{1}<b_{1} \leq-a_{1}$. Let $c_{1}=\left(b_{1}^{2}+d\right) /\left(4 a_{1}\right)$ and observe that $c_{1} \geq d /\left(4 a_{1}\right)>a_{1}$ using (3.1). From (1.2) we see that the quadratic form $Q=a_{1} x^{2}+b_{1} x y+c_{1} y^{2}$ is reduced. 
Lemma 2. Let a satisfy $2 \leq a \leq(d / 4)^{1 / 2}$ and $\operatorname{gcd}(a, d)=1$. Then $a \in M_{d}$ if and only if every prime divisor $p$ of a satisfies $(-d \mid p)=1$.

Proof. First, assume that $a \in M_{d}$. Since $b^{2}-4 a c=-d$, we see that

$$
b^{2} \equiv-d(\bmod 4 a)
$$

If $2 \mid a$, then we see at once that $b^{2} \equiv-d(\bmod 8)$. We know that $-d$ must be odd because $a$ is even and, by hypothesis, $\operatorname{gcd}(a, d)=1$. Since 1 is the only odd square modulo 8 , we have $d \equiv-1(\bmod 8)$. Thus $(-d \mid 2)=1$. If $p$ is any odd prime dividing $a$, it follows at once from $(3.2)$ that $b^{2} \equiv-d$ $(\bmod p)$. Thus $(-d \mid p)=1$, and the "only if" direction is proved.

Conversely, assume that $(-d \mid p)=1$ for each prime divisor $p$ of $a$. If $p$ is odd, then the congruence $z^{2} \equiv-d(\bmod p)$ is solvable, and by Hensel's lemma, the congruence $z^{2} \equiv-d\left(\bmod p^{\alpha}\right)$ is solvable for all $\alpha \in \mathbb{Z}^{+}$. If $p=2$, note that $(-d \mid 2)=1$ implies $d \equiv-1(\bmod 8)$. Hence, the congruence $z^{2} \equiv-d(\bmod 8)$ is solvable. Furthermore, it is well known that solutions with $z \equiv \pm 1(\bmod 8)$ can be lifted to a solution of the congruence $z^{2} \equiv-d$ $\left(\bmod 2^{\alpha}\right)$ for all $\alpha \in \mathbb{Z}^{+}$. Hence, by the Chinese Remainder Theorem, there exists a $b$ such that the congruence $b^{2} \equiv-d(\bmod 4 a)$ is solvable. Reasoning as in the proof of Lemma 1 , there is in fact such a $b$ with $-a<b \leq a$. Let $c=\left(b^{2}+d\right) /(4 a)$. If $b \neq 0$, then $c>d /(4 a) \geq a$ since $a \leq(d / 4)^{1 / 2}$ by hypothesis. If $b=0$, then $c=d /(4 a) \geq a$. In every case, $a x^{2}+b x y+c y^{2}$ is reduced, and $a \in M_{d}$.

Lemma 3. Suppose $h(-d)$ is odd. If $a>1$ and $a x^{2}+b x y+c y^{2} \in Q_{d}$, then $a x^{2}-b x y+c y^{2}$ is a distinct member of $Q_{d}$.

Proof. Since $a>1$ and $a \in M_{d}$, we know that $h(-d)>1$. Hence, $(2.1)$ implies that $d$ is prime. Since $a x^{2}+b x y+c y^{2}$ is a reduced form, we know that $a x^{2}-b x y+c y^{2}$ will be a distinct reduced form unless (i) $b=0$, (ii) $b=a$, or (iii) $a=c$. If (i) is true, then $4 a c=d$, contradicting the fact that $d$ is prime. If (ii) is true, then $a^{2}-4 a c=a(a-4 c)=-d$. Since $a>1$ and $d$ is prime, we have $a=d$, which is impossible since $a<(d / 3)^{1 / 2}$. If (iii) is true, then $b^{2}-4 a^{2}=(b-2 a)(b+2 a)=-d$. Since $b \geq 0$ in case (iii) and $d$ is prime, we know that $2 a-b=a+(a-b)=1$. This leads to a contradiction since $a>1$ by hypothesis and $a \geq b$ by (1.3).

Lemma 4. Suppose $h(-d)$ is odd. Let $a \in \mathbb{Z}^{+}$be odd and satisfy $a \leq(d / 4)^{1 / 2}$. If $a \in M_{d}$, then a appears in $M_{d}$ exactly $2^{\omega(a)}$ times.

Proof. If $a=1$, it is easy to see from (1.2) that $a$ appears precisely once in $M_{d}$. Thus, we may henceforth assume that $a>1$. Of course, this implies that $h(-d)>1$ as well. We want to count the number of integers $b$ such that $a x^{2}+b x y+\left(\left(b^{2}+d\right) /(4 a)\right) y^{2} \in Q_{d}$. By (1.2) this is just the 
number of integers $b$ that satisfy $\left(b^{2}+d\right) /(4 a) \in \mathbb{Z}$ with either $-a<b \leq a<$ $\left(b^{2}+d\right) /(4 a)$ or $0 \leq b \leq a=\left(b^{2}+d\right) /(4 a)$. Assume, for the moment, that $\left(b^{2}+d\right) /(4 a) \in \mathbb{Z}$. Since $h(-d)>1$, we see from $(2.1)$ that $d \equiv 3(\bmod 4)$. It follows at once that $b$ is nonzero. Using the hypothesis, $a \leq(d / 4)^{1 / 2}$, we then deduce $a \leq d /(4 a)<\left(b^{2}+d\right) /(4 a)$. It follows that we want to count the number of integers $b$ that satisfy $b^{2} \equiv-d(\bmod 4 a)$ with $-a<b \leq a$.

From (2.1), we know that $d$ is prime. Since $a \leq(d / 4)^{1 / 2}<d$, we see that $\operatorname{gcd}(a, d)=1$. Let $p$ be any prime divisor of $a$. By Lemma 2, we see that $(-d \mid p)=1$. Since $a$ is odd, we know that $p$ is also. Hence, the congruence $z^{2} \equiv-d(\bmod p)$ has exactly two solutions. By Hensel's Lemma, we know that the congruence $z^{2} \equiv-d\left(\bmod p^{\alpha}\right)$ has exactly two solutions for all $\alpha \in \mathbb{Z}^{+}$. Also, since $d \equiv 1(\bmod 4)$, we know that the congruence $z^{2} \equiv$ $-d(\bmod 4)$ has exactly two solutions. Thus by the Chinese Remainder Theorem, we see that there are precisely $2^{\omega(a)+1}$ integers $b$ that satisfy both

$$
b^{2} \equiv-d(\bmod 4 a)
$$

and $-2 a<b \leq 2 a$. Now either both $b$ and $b+2 a$ satisfy (3.3) or neither do. Hence, the number of $b$ that satisfy (3.3) with $-2 a<b \leq-a$ is equal to the number of $b$ that satisfy (3.3) with $0<b \leq a$. A similar argument with $b$ and $b-2 a$ shows that the number of $b$ that satisfy (3.3) with $a<b \leq 2 a$ is equal to the number of $b$ that satisfy (3.3) with $-a<b \leq 0$. It follows that exactly $2^{\omega(a)}$ integers $b$ satisfy (3.3) with $-a<b \leq a$, thereby proving Lemma 4.

Lemma 5. If $a>1, a \in M_{d}$ and $(a, d)=1$, then $a>(d / 4)^{1 / h(-d)}$.

Proof. Suppose $p \mid a$ and $p$ is an odd prime. Then $b^{2} \equiv-d(\bmod p)$ implies $(-d \mid p)=1$. Also, if $2 \mid a$, then $b^{2} \equiv-d(\bmod 8)$. Hence, $-d \equiv 1$ $(\bmod 8)$, so that $(-d \mid 2)=1$. Thus, if $p$ is any prime dividing $a$ we have $(-d \mid p)=1$. This implies that $p$ splits in $\mathbb{Q}(\sqrt{-d}),\langle p\rangle=\wp_{1} \wp_{2}$ with $\wp_{1} \neq \wp_{2}$. Thus, $\wp_{1}^{h(-d)}=\langle\beta\rangle$ is a principal ideal, and $\beta \notin \mathbb{Z}$. It follows that $p^{h(-d)}=$ $N\left(\wp_{1}^{h(-d)}\right)=N(\langle\beta\rangle) \geq d / 4$. Since $a \geq p$, it follows that $a \geq(d / 4)^{1 / h(-d)}$. Note that $a>1$ implies $h(-d)>1$, so that $(d / 4)^{1 / h(-d)} \notin \mathbb{Z}$. The lemma follows.

It follows from (1.3), (2.1) and Lemma 5 that

$$
\left(\frac{d}{4}\right)^{1 / h(-d)}<a \leq\left(\frac{d}{3}\right)^{1 / 2} \quad\left(a \in M_{d} \backslash\{1\}, h(-d)>1 \text { is odd }\right) .
$$

To improve on these bounds, we separate the minima to a certain extent using the multiplicative structure of $M_{d}$ developed in Lemmas 1-4.

Assume that $h(-d)>1$ and $h(-d)$ is odd. From Lemma 3 we know that every $a \in M_{d} \backslash\{1\}$ appears an even number of times in $M_{d}$. Also, by (3.4) 
if $d>2^{h(-d)+2}$, then 2 is not a minimum. It then follows by Lemma 1 that $a$ must be odd if $a \in M_{d}$. In the remainder of this section assume that $d>2^{h(-d)+2}$. Define the multiset $M_{d}^{*}$ by

$$
M_{d}^{*}=\left\{a: a x^{2}+b x y+c y^{2} \in Q_{d}, a>1, b>0\right\} .
$$

From Lemma 3 we have

$$
\left|M_{d}^{*}\right|=(h(-d)-1) / 2 .
$$

Definition 1. A partition for $d$ is a list of $(h(-d)-1) / 2$ pairs of functions $\left(l_{a}(d), u_{a}(d)\right)$, each increasing in $d$, corresponding to the $(h(-d)-1) / 2$ elements in $M_{d}^{*}$.

DeFinition 2. We say a partition for $d$ covers $M_{d}$ if

$$
l_{a}(d) \leq a \leq u_{a}(d)
$$

for every $a \in M_{d}^{*}$.

We begin with a simple example. Suppose that $h(-d)=5$. Let $p$ be the smallest nonprincipal member of $M_{d}$. By Lemma 1, $p$ is prime. In past investigations of minima one generally used the result contained in Lemma 5 , which states that all minima $a$, except $a=1$, must satisfy $a>(d / 4)^{1 / 5}$. In particular, the minima could simultaneously be small, each minimum lying close to the bound $(d / 4)^{1 / 5}$. However, using Lemma 2 and Lemma 4, we see that if $p$ is about $(d / 4)^{1 / 5}$ in size, then there are exactly two reduced forms with minimum $p$ and two reduced forms with minimum $p^{2}$ and one reduced form with minimum 1, which provides us with five reduced forms. This excludes the possibility that any other minimum is simultaneously small. Using similar reasoning it is not hard to see that if $h(-d)=5$, then $M_{d}$ is covered by one of the following three partitions:

$$
\begin{array}{lll}
1 & (d / 4)^{1 / 5} \leq p \leq(d / 4)^{1 / 4} & (d / 4)^{2 / 5} \leq p^{2} \leq(d / 4)^{1 / 2} \\
2 & (d / 4)^{1 / 4} \leq p \leq(d / 4)^{1 / 3} & (d / 4)^{1 / 4} \leq a \leq(d / 3)^{1 / 2} \\
3 & (d / 4)^{1 / 3} \leq a \leq(d / 3)^{1 / 2} & (d / 4)^{1 / 3} \leq a \leq(d / 3)^{1 / 2}
\end{array}
$$

For each fixed value of $m=5,7, \ldots, 23$, a set of partitions covering all possible $M_{d}$ is given in Appendix B. In these tables, $p$ and $q$ denote the first and second smallest prime minima in $M_{d}$, respectively, while $a$ denotes a generic member of $M_{d}, v=d / 4$ and $w=d / 3$. In order to simplify our presentation of a covering partition, we now introduce some additional notation. The notation (3p.), for example, denotes that the inequalities for the first three powers of the prime are trivially inferred. Similarly, the notation (3), for example, means the inequality is to be listed three times. In this 
notation, the covering partitions for $M_{d}$ when $h(-d)=5$ are given by

$$
\begin{array}{ll}
1 & v^{1 / 5} \leq p \leq v^{1 / 4}(2 \mathrm{p} .) \\
2 & v^{1 / 4} \leq p \leq v^{1 / 3} \\
3 & v^{1 / 3} \leq a \leq w^{1 / 2}(2)
\end{array} \quad v^{1 / 4} \leq a \leq w^{1 / 2}
$$

In order to give a better idea of how the partitions in Appendix B were generated, we will go through the details of partition number 10 for class number 23 (Table B10). The partition is given by

$$
\begin{aligned}
& 10 \quad v^{1 / 10} \leq p \leq v^{1 / 8}(4 \mathrm{p} .) \quad v^{3 / 16} \leq q \leq v^{1 / 4}(2 \mathrm{p} .) \quad v^{23 / 80} \leq p q \leq v^{3 / 8} \\
& v^{31 / 80} \leq p^{2} q \leq v^{1 / 2}(2) \quad v^{3 / 8} \leq a \leq w^{1 / 2}
\end{aligned}
$$

Assume the smallest nonprincipal minimum $p$ satisfies $v^{1 / 10} \leq p \leq v^{1 / 8}$; then $p^{2}, p^{3}, p^{4}$ are all $\leq(d / 4)^{1 / 2}$, implying that $p, p^{2}, p^{3}, p^{4} \in M_{d}$, which accounts for 8 minima. If the second smallest minimum $q$ satisfies $q \leq$ $(d / 4)^{3 / 16}$, then $q, q^{2}, p q, p q^{2}, p^{2} q$ are $\leq(d / 4)^{1 / 2}$, implying that they are in $M_{d}$. But this accounts for 16 new minima, which would make 25 total. Thus, $v^{3 / 16} \leq q$. Assume $q \leq(d / 4)^{1 / 4}$. Now, $q, q^{2}, p q, p^{2} q \leq(d / 4)^{1 / 2}$, so we have accounted for 21 minima. If any further minima $a$ satisfy $a \leq(d / 4)^{3 / 8}$, then $p a \leq(d / 4)^{1 / 2}$ would imply that there are more than 23 minima. Therefore, the remaining two minima must satisfy $(d / 4)^{3 / 8} \leq a \leq(d / 3)^{1 / 2}$. All of the partitions in the tables of Appendix B are computed in this same fashion. By using a disjoint set of assumptions on the first and second largest prime minima $p$ and $q$, these sets of partitions can be seen to cover all possible $M_{d}$.

4. Properties of an auxiliary function. In this section we prove two technical lemmas concerning the auxiliary function $F$ defined by

$$
F(x)=\sqrt{x} \sum_{n=1}^{\infty} d(n) K_{0}(n x) \quad(x>0),
$$

where $K_{0}(z)$ is defined in (1.4).

On several occasions in $\S 5.2$ and $\S 5.3$ we will need to compute accurate approximations to $F$ at certain small arguments. For such purposes, the following crude generalization of [18, Lemma 7]) suffices.

LEMma 6. If $x>0, N$ is a nonnegative integer, and

$$
\Delta_{N}(x)=F(x)-\sqrt{x} \sum_{n=1}^{N} d(n) K_{0}(n x),
$$

then

$$
\left|\Delta_{N}(x)\right| \leq \frac{2}{\sqrt{x}}(1+\log (N+1+2 / x)) e^{-(N+1) x / 2}
$$

where the empty sum is understood to equal zero when $N=0$. 
Proof. Since $x>0$, we see from (4.1) and (1.4) that

$$
0 \leq x^{-1 / 2} \Delta_{N}(x) \leq \sum_{n=N+1}^{\infty} d(n) \int_{1}^{\infty} e^{-n x t / 2} \frac{d t}{t}=\sum_{n=N+1}^{\infty} d(n) \int_{n x / 2}^{\infty} e^{-u} \frac{d u}{u} .
$$

Using partial summation, we have

$$
x^{-1 / 2} \Delta_{N}(x) \leq \int_{(N+1) x / 2}^{\infty} e^{-u}\left(\sum_{n \leq 2 u / x} d(n)\right) \frac{d u}{u} .
$$

For $y \geq 1$ we have

$$
\sum_{n \leq y} d(n)=\sum_{n \leq y}\left\lfloor\frac{y}{n}\right\rfloor \leq y \sum_{n \leq y} \frac{1}{n} \leq y(1+\log y)
$$

so that

$$
\left|x^{-1 / 2} \Delta_{N}(x)\right| \leq \int_{(N+1) x / 2}^{\infty} e^{-u} \frac{2 u}{x}(1+\log (2 u / x)) \frac{d u}{u} .
$$

An integration by parts gives

$$
\left|x^{-1 / 2} \Delta_{N}(x)\right| \leq \frac{2}{x}\left((1+\log (N+1)) e^{-(N+1) x / 2}+\int_{(N+1) x / 2}^{\infty} e^{-u} \frac{d u}{u}\right) .
$$

Note that the function $g$, defined by

$$
g(v)=\int_{v}^{\infty} e^{-u} \frac{d u}{u}-e^{-v} \log (1+1 / v)
$$

is increasing for $v>0$, and $\lim _{v \rightarrow \infty} g(v)=0$. Hence,

$$
\int_{(N+1) x / 2}^{\infty} e^{-u} \frac{d u}{u} \leq e^{-(N+1) x / 2} \log \left(1+\frac{2}{(N+1) x}\right)
$$

and the lemma follows.

The next lemma will play an important role in $\S 5.2$ and $\S 5.3$.

Lemma 7. $F(x)$ is a strictly decreasing function of $x$ for $x>0$.

Proof. From (4.1) we have

$$
F(x)=\sqrt{x} \sum_{n=1}^{\infty} d(n) K_{0}(n x)=\sqrt{x} \sum_{n=1}^{\infty} \sum_{d \mid n} K_{0}(n x)=\sqrt{x} \sum_{d=1}^{\infty} \sum_{m=1}^{\infty} K_{0}(d m x),
$$

where $K_{0}$ is the modified Bessel function of the second kind of order zero 
given by (1.4). Hence, we get

$$
\begin{aligned}
F(x) & =\sqrt{x} \int_{1}^{\infty}\left(\sum_{d=1}^{\infty} \sum_{m=1}^{\infty} \exp \left(-\frac{d m x}{2}\left(t+t^{-1}\right)\right)\right) \frac{d t}{t} \\
& =\int_{1}^{\infty}\left(\sum_{d=1}^{\infty} \frac{\sqrt{x}}{\exp \left(\frac{d\left(t+t^{-1}\right)}{2} x\right)-1}\right) \frac{d t}{t} .
\end{aligned}
$$

For $b>0$, define

$$
f_{b}(x)=\frac{\sqrt{x}}{e^{b x}-1}
$$

Since $x \mapsto x /\left(e^{x b}-1\right)$ decreases on $(0, \infty)$, we know that $x \mapsto f_{b}(x)$ also decreases on $(0, \infty)$. Lemma 7 follows immediately.

5. The medium range. Let $\chi$ be a real primitive character modulo $k$ for some integer $k>1$. In the case where $-k$ is a negative fundamental discriminant we take $\chi(n)=(-k \mid n)$ (see [7, p. 40]). Define

$$
A_{d}(s)=\sum_{Q_{d}} \chi(a) a^{-s}
$$

and

$$
P_{k}(s)=\prod_{p \mid k}\left(1-p^{-s}\right)
$$

In 1966 Stark [22] exploited a formula for the zeta function of a quadratic number field (i.e., $\left.\zeta(s) L\left(s, \chi_{1}\right)\right)$ to show that if a tenth fundamental discriminant $-d$ of class number 1 existed, then $d>\exp \left(2.2 \cdot 10^{7}\right)$. Later Stark [25] developed a formula for $L(s, \chi) L\left(s, \chi \chi_{1}\right)$ analogous to the formula for $\zeta(s) L\left(s, \chi_{1}\right)$. Montgomery and Weinberger [18] exploited this formula to obtain similar results for class numbers 2 and 3 . Indeed, if $(k, d)=1$, then

$$
\left(\frac{k \sqrt{d}}{2 \pi}\right)^{s-1 / 2} \Gamma(s) L(s, \chi) L\left(s, \chi \chi_{1}\right)=T_{d}(s)+T_{d}(1-s)+U_{d}(s)
$$

where

$$
\begin{gathered}
T_{d}(s)=\left(\frac{k \sqrt{d}}{2 \pi}\right)^{s-1 / 2} \Gamma(s) \zeta(2 s) P_{k}(2 s) A_{d}(s), \\
U_{d}(s)=\frac{4 \sqrt{\pi}}{k} \sum_{Q_{d}} a^{-1 / 2} \sum_{n=1}^{\infty} K_{s-1 / 2}\left(\frac{\pi n \sqrt{d}}{a k}\right) n^{s-1 / 2} V_{Q}(s, n),
\end{gathered}
$$

and

$$
V_{Q}(s, n)=\sum_{y \mid n} y^{1-2 s} \Re\left\{\sum_{j=1}^{k} \chi(Q(j, y)) e\left(\frac{j n}{k y}\right) e\left(\frac{b n}{2 a k}\right)\right\} .
$$


Let $s_{0}=1 / 2+i t_{0}$, with $t_{0}>0$, be a zero of $L(s, \chi)$. Substituting $s=s_{0}$ into (5.1) gives

$$
T_{d}\left(s_{0}\right)+T_{d}\left(\bar{s}_{0}\right)=-U_{d}\left(s_{0}\right) .
$$

Applying the Schwarz Reflection Principle to $T_{d}$ implies

$$
2\left|T_{d}\left(s_{0}\right)\right| \cos \left(\arg T_{d}\left(s_{0}\right)\right)=-U_{d}\left(s_{0}\right),
$$

which, in turn, gives

$$
\left|\sin \arg \left(i T_{d}\left(s_{0}\right)\right)\right|=\left|\frac{U_{d}\left(s_{0}\right)}{2 T_{d}\left(s_{0}\right)}\right| .
$$

The method for the middle range consists in showing that this equality is false for large intervals of $d$ under the assumption that $h(-d)$ is some fixed odd integer. Indeed, for fixed $k$ and $t_{0}$, define the constants

(5.4) $\xi_{1}=t_{0} / 2$,

$$
\begin{aligned}
& \xi_{2}=t_{0} \log \left(\frac{k}{2 \pi}\right)+\arg \left\{i \Gamma\left(1 / 2+i t_{0}\right) \zeta\left(1+2 i t_{0}\right) P_{k}\left(1+2 i t_{0}\right)\right\} \\
& \xi_{3}=2\left|\Gamma\left(1 / 2+i t_{0}\right) \zeta\left(1+2 i t_{0}\right) P_{k}\left(1+2 i t_{0}\right)\right| .
\end{aligned}
$$

To show (5.3) is false, all we need to show is that

$$
\left|\sin \left(\xi_{1} \log d+\xi_{2}+\arg A_{d}\left(s_{0}\right)\right)\right|>\frac{\left|U_{d}\left(s_{0}\right)\right|}{\xi_{3}\left|A_{d}\left(s_{0}\right)\right|} .
$$

5.1. The range $d_{2}(m) \leq d \leq d_{3}(m)$

Lemma 8. Let $t \in \mathbb{R}^{+}$. Suppose $m=h(-d)$ is odd and fixed. Then

$$
\left|A_{d}(1 / 2+i t)\right| \geq 1-\frac{m-1}{(d / 4)^{1 /(2 m)}} .
$$

Furthermore, if $d>\max \left\{4 e^{2 m}, 4(m-1)^{2 m}\right\}$, then

$$
\left|\arg A_{d}(1 / 2+i t)\right| \leq \frac{t(1-1 / m) \log (d / 4)}{(d / 4)^{1 /(2 m)}-(m-1)} .
$$

Proof. Both (5.8) and (5.9) are trivially true if $m=1$, so assume $m>1$. Using the lower bound in (3.4) gives

$$
\begin{aligned}
\left|A_{d}(1 / 2+i t)-1\right| & =\left|\sum_{Q_{d}, a \neq 1} \chi(a) a^{-1 / 2-i t}\right| \\
& \leq \sum_{Q_{d}, a \neq 1} a^{-1 / 2} \\
& \leq \frac{m-1}{(d / 4)^{1 /(2 m)}} .
\end{aligned}
$$

Now, (5.8) follows from (5.11) and the triangle inequality. 
For the remainder of the proof assume $d>\max \left\{4 e^{2 m}, 4(m-1)^{2 m}\right\}$. Since $d>4(m-1)^{2 m}$, we know from (5.11) that

$$
\left|A_{d}(1 / 2+i t)-1\right|<1,
$$

so that $A_{d}(1 / 2+i t)$ lies in the right half plane. Hence, when we write $\arg A_{d}(1 / 2+i t)$ in (5.9) and below, we can, without loss of generality, assume we are dealing with the principal value of the argument. Let $L$ be the line segment joining $1 / 2$ to $1 / 2+i t$. Then an equation for $L$ is given by $\ell(u)=1 / 2+i u, 0 \leq u \leq t$. Since $d>4(m-1)^{2 m}$, we know by (5.8) that $A_{d}$ does not vanish on $L$. Furthermore, $A_{d}(s)$ is an entire function of $s$. It follows $[17$, p. 218] that

$$
\int_{L} \frac{A_{d}^{\prime}(z)}{A_{d}(z)} d z=\int_{0}^{t} \frac{A_{d}^{\prime}(1 / 2+i u)}{A_{d}(1 / 2+i u)} i d u=\left.\log A_{d}(1 / 2+i u)\right|_{0} ^{t} .
$$

Evaluating the right-hand side and taking imaginary parts yields

$$
\begin{aligned}
\left|\arg A_{d}(1 / 2+i t)\right| & =\left|\Im \int_{0}^{t} \frac{A_{d}^{\prime}(1 / 2+i u)}{A_{d}(1 / 2+i u)} i d u\right| \\
& \leq t \max _{0 \leq u \leq t}\left|\frac{A_{d}^{\prime}(1 / 2+i u)}{A_{d}(1 / 2+i u)}\right|
\end{aligned}
$$

Note that

$$
\begin{aligned}
\left|A_{d}^{\prime}(1 / 2+i t)\right| & =\left|\sum_{Q_{d}, a \neq 1} \chi(a) a^{-1 / 2-i t} \log a\right| \\
& \leq \sum_{Q_{d}, a \neq 1} a^{-1 / 2} \log a .
\end{aligned}
$$

Now, $x^{-1 / 2} \log x$ is a decreasing function of $x$ for $x>e^{2}$. Using (3.4) and the hypothesis $d>4 e^{2 m}$, it then follows from (5.13) that

$$
\left|A_{d}^{\prime}(1 / 2+i t)\right| \leq \frac{(1-1 / m) \log (d / 4)}{(d / 4)^{1 /(2 m)}} .
$$

Using (5.14) and (5.8) in (5.12) yields (5.9), and the lemma is proved.

Lemma 9. Let $t \in \mathbb{R}$. If $k>1$ is an odd square-free integer, then

$$
\left|U_{d}(1 / 2+i t)\right| \leq \frac{4}{d^{1 / 4}} \sum_{r \mid k} \frac{3^{\omega(r)} 2^{\omega(k)-\omega(r)}}{r^{1 / 2}} \sum_{a \in M_{d}} F\left(\frac{\pi \sqrt{d} r^{2}}{a k}\right) .
$$

Proof. From the definition of $U_{d}$ in (5.2) we deduce at once that

$$
\left|U_{d}(1 / 2+i t)\right| \leq \frac{4 \sqrt{\pi}}{k} \sum_{Q_{d}} a^{-1 / 2} \sum_{n=1}^{\infty} K_{0}\left(\frac{\pi \sqrt{d} n}{a k}\right)\left|V_{Q}(1 / 2+i t, n)\right| .
$$


Using an argument of Weil [30, his inequality (5)], Montgomery and Weinberger [18, Lemma 7] have shown that

$$
\begin{aligned}
\left|V_{Q}(1 / 2+i t, n)\right| & \leq 2^{\omega(k)} k^{1 / 2} \sum_{y \mid n} \prod_{p \mid(y, n / y, k)} \frac{p^{1 / 2}}{2} \\
& =2^{\omega(k)} k^{1 / 2} \sum_{r \mid k} 2^{-\omega(r)} r^{1 / 2} \sum_{\substack{y \mid n \\
(y, n / y, k)=r}} 1,
\end{aligned}
$$

because $k$ is square-free. Since $(y, n / y, k)=r$ implies $r^{2} \mid n$, we have

$$
\left|V_{Q}(1 / 2+i t, n)\right| \leq 2^{\omega(k)} k^{1 / 2} \sum_{\substack{r\left|k \\ r^{2}\right| n}} 2^{-\omega(r)} r^{1 / 2} d(n) .
$$

Inserting this into (5.16) gives

$$
\begin{aligned}
& \left|U_{d}(1 / 2+i t)\right| \\
& \quad \leq \frac{4 \sqrt{\pi}}{k^{1 / 2}} \sum_{a \in M_{d}} \frac{1}{a^{1 / 2}} \sum_{r \mid k} 2^{\omega(k)-\omega(r)} r^{1 / 2} \sum_{\substack{n=1 \\
r^{2} \mid n}}^{\infty} d(n) K_{0}\left(\frac{\pi \sqrt{d} n}{a k}\right) .
\end{aligned}
$$

Let $n=r^{2} m$ and note that $d(n) \leq d\left(r^{2}\right) d(m)=3^{\omega(r)} d(m)$ since $r$ is squarefree. From (5.17) we have

$$
\begin{aligned}
& \left|U_{d}(1 / 2+i t)\right| \\
& \quad \leq \frac{4 \sqrt{\pi}}{k^{1 / 2}} \sum_{a \in M_{d}} \frac{1}{a^{1 / 2}} \sum_{r \mid k}\left(\frac{3}{2}\right)^{\omega(r)} 2^{\omega(k)} r^{1 / 2} \sum_{m=1}^{\infty} d(m) K_{0}\left(\frac{\pi \sqrt{d} r^{2} m}{a k}\right) .
\end{aligned}
$$

Applying definition (4.1) to the inner sum gives the result.

Corollary. Let $t \in \mathbb{R}$ and $m=h(-d)$. If $k>1$ is an odd square-free integer, then

$$
\left|U_{d}(1 / 2+i t)\right| \leq \frac{8 k^{1 / 2} \log k}{3^{1 / 4} \pi^{1 / 2} d^{1 / 4}}\left(m-1+e^{-\pi\left(d^{1 / 2}-\sqrt{3}\right) /(2 k)}\right) \prod_{p \mid k}\left(2+3 p^{-3 / 2}\right) .
$$

P r o of. Replace $F$ in Lemma 9 with the upper bound given in Lemma 6 with $N=0$ to get

$$
\begin{aligned}
\left|U_{d}(1 / 2+i t)\right| \leq & \frac{8 k^{1 / 2}}{\pi^{1 / 2} d^{1 / 2}} \sum_{r \mid k}\left(\frac{3}{2}\right)^{\omega(r)} 2^{\omega(k)} r^{-3 / 2} \\
& \times \sum_{a \in M_{d}} a^{1 / 2}\left(1+\log \left(1+\frac{2 a k}{\pi d^{1 / 2} r^{2}}\right)\right) e^{-\pi d^{1 / 2} r^{2} /(2 a k)}
\end{aligned}
$$


In the inner sum, the $a=1$ term is treated separately. When $a>1$ we use the inequality $a \leq(d / 3)^{1 / 2}$. In both cases, we also use the inequalities $r \geq 1, d \geq 3$, and

$$
\left(1+\log \left(1+\frac{2 k}{\pi \sqrt{3}}\right)\right) e^{-\pi \sqrt{3} /(2 k)} \leq \log k \quad(k \geq 2)
$$

to finish the proof of the corollary.

For the remainder of $\S 5.1$, we assume there exists a discriminant $-d$ with the following properties:

(I) $h(-d)=m$, where $m \in\{5,7,9, \ldots, 23\}$ is fixed;

(II) $d_{2}(m) \leq d \leq 10^{850}$, where $d_{2}(m)$ is given in Table 3 near the end of this subsection.

Our goal is to show that (5.7) is true for $d$ with a suitable choice of $k$ and $s_{0}$. For this purpose, we need a small zero, $s_{0}=1 / 2+i t_{0}$, of $L(s, \chi)$. Weinberger [31] has computed several such zeros, each corresponding to a different value of $k$. In this section, we use

$$
k=115147 \text { and } t_{0}=0.003157614
$$

where the absolute error in $t_{0}$ is less than $10^{-8}$, but we only make use of the first 4 significant places. From (5.4)-(5.6) we then have

$$
\xi_{1}=0.001579, \quad \xi_{2}=0.02875, \quad \xi_{3}=555.8,
$$

where these approximations are accurate to the number of places shown.

Since $m \leq 23$ and $d \geq d_{2}(m) \geq 10^{63}$ by Table 3 , the right-hand side of (5.8) is positive. Hence, letting $t=t_{0}$ in Lemma 8, we see from (5.8) that $\left|A_{d}\left(s_{0}\right)\right|$ does not vanish. Thus, using (5.8) and the corollary to Lemma 9 with $t=t_{0}$, we have

where

$$
\frac{\left|U_{d}\left(s_{0}\right)\right|}{\xi_{3}\left|A_{d}\left(s_{0}\right)\right|} \leq R_{2}(d)
$$

$$
R_{2}(d)=\frac{8 k^{1 / 2} \log k\left(m-1+e^{-\pi\left(d^{1 / 2}-\sqrt{3}\right) /(2 k)}\right) \prod_{p \mid k}\left(2+3 p^{-3 / 2}\right)}{\xi_{3} 3^{1 / 4} \pi^{1 / 2} d^{1 / 4}\left(1-(m-1) /(d / 4)^{1 /(2 m)}\right)} .
$$

Clearly $R_{2}(d)$ is decreasing in $d$ so that

$$
\frac{\left|U_{d}\left(s_{0}\right)\right|}{\xi_{3}\left|A_{d}\left(s_{0}\right)\right|} \leq R_{2}\left(d_{2}(m)\right)
$$

since $d \geq d_{2}(m)$. Upper bounds for $R_{2}\left(d_{2}(m)\right)$ are given in Table 3 .

Since $5 \leq m \leq 23$ we have $\max \left\{4 e^{2 m}, 4(m-1)^{2 m}\right\}=4(m-1)^{2 m}<$ $10^{63}<d_{2}(m)$. Hence, all of the hypotheses of Lemma 8 hold for $t=t_{0}$ and $m \in\{5,7, \ldots, 23\}$. We deduce from (5.9) that

$$
\left|\arg A_{d}\left(s_{0}\right)\right| \leq \alpha_{2}(d),
$$


where

$$
\alpha_{2}(d)=\frac{t(1-1 / m) \log (d / 4)}{(d / 4)^{1 /(2 m)}-(m-1)} .
$$

It is easy to see that $\alpha_{2}(d)$ is decreasing for $d \geq 4 e$, so we certainly have

$$
\left|\arg A_{d}\left(s_{0}\right)\right| \leq \alpha_{2}\left(d_{2}(m)\right)
$$

since $d \geq d_{2}(m)$. Upper bounds for $\alpha_{2}\left(d_{2}(m)\right)$ are given in Table 3 .

Let $\beta_{2}(m)$ be defined by

$$
\beta_{2}(m)=\xi_{1} \log d_{2}(m)+\xi_{2}-\alpha_{2}\left(d_{2}(m)\right)
$$

and $\gamma_{2}(m)$ be defined by

$$
\gamma_{2}(m)=\xi_{1} \log 10^{850}+\xi_{2}+\alpha_{2}\left(d_{2}(m)\right) .
$$

Lower bounds for $\beta_{2}(m)$ and upper bounds for $\gamma_{2}(m)$ are given in Table 3 . Using Table 3 , the fact that $d_{2}(m) \leq d$, and (5.19), we have

$$
0<\beta_{2}(m) \leq \xi_{1} \log d+\xi_{2}+\arg A_{d}\left(s_{0}\right) .
$$

Using the fact that $d \leq 10^{850},(5.19)$, and Table 3 , we have

$$
\xi_{1} \log d+\xi_{2}+\arg A_{d}\left(s_{0}\right) \leq \gamma_{2}(m)<\pi .
$$

Hence,

$$
\left|\sin \left(\xi_{1} \log d+\xi_{2}+\arg A_{d}\left(s_{0}\right)\right)\right| \geq \min \left\{\left|\sin \beta_{2}(m)\right|,\left|\sin \gamma_{2}(m)\right|\right\} .
$$

Lower bounds for $\left|\sin \beta_{2}(m)\right|$ and $\left|\sin \gamma_{2}(m)\right|$ are given in Table 3 .

From (5.20) and Table 3 we deduce that

$$
\left|\sin \left(\xi_{1} \log d+\xi_{2}+\arg A_{d}\left(s_{0}\right)\right)\right|>R_{2}\left(d_{2}(m)\right) .
$$

In light of (5.18), (5.7) follows immediately. Since (5.3) is false, we conclude that $h(-d) \neq m$ for $m \in\{5,7, \ldots, 23\}$ and $d_{2}(m) \leq d \leq 10^{850}$. Note that from Table 2 in $\S 2$, we have certainly covered the range $d_{2}(m) \leq d \leq d_{3}(m)$.

Table 3. $h(-d) \neq m$ for $d_{2}(m) \leq d \leq 10^{850}$

\begin{tabular}{rlcccccc}
\hline$m$ & $d_{2}(m)$ & $R_{2}\left(d_{2}(m)\right)$ & $\alpha_{2}\left(d_{2}(m)\right)$ & $\beta_{2}(m)$ & $\left|\sin \beta_{2}(m)\right|$ & $\gamma_{2}(m)$ & $\left|\sin \gamma_{2}(m)\right|$ \\
\hline 5 & $10^{65}$ & $2.2 \cdot 10^{-14}$ & $1.4 \cdot 10^{-7}$ & 0.264 & 0.26 & 3.121 & 0.020 \\
7 & $10^{65}$ & $3.3 \cdot 10^{-14}$ & $1.1 \cdot 10^{-5}$ & 0.264 & 0.26 & 3.121 & 0.020 \\
9 & $10^{66}$ & $2.5 \cdot 10^{-14}$ & $9.9 \cdot 10^{-5}$ & 0.268 & 0.26 & 3.121 & 0.020 \\
11 & $10^{68}$ & $9.9 \cdot 10^{-15}$ & $3.9 \cdot 10^{-4}$ & 0.275 & 0.27 & 3.121 & 0.020 \\
13 & $10^{73}$ & $6.8 \cdot 10^{-16}$ & $8.2 \cdot 10^{-4}$ & 0.293 & 0.28 & 3.121 & 0.020 \\
15 & $10^{76}$ & $1.5 \cdot 10^{-16}$ & $1.7 \cdot 10^{-3}$ & 0.303 & 0.29 & 3.122 & 0.019 \\
17 & $10^{79}$ & $3.1 \cdot 10^{-17}$ & $2.9 \cdot 10^{-3}$ & 0.312 & 0.30 & 3.124 & 0.017 \\
19 & $10^{79}$ & $3.8 \cdot 10^{-17}$ & $5.6 \cdot 10^{-3}$ & 0.310 & 0.30 & 3.126 & 0.015 \\
21 & $10^{79}$ & $4.8 \cdot 10^{-17}$ & $1.1 \cdot 10^{-2}$ & 0.304 & 0.29 & 3.132 & 0.009 \\
23 & $10^{79}$ & $6.8 \cdot 10^{-17}$ & $2.0 \cdot 10^{-2}$ & 0.295 & 0.29 & 3.141 & $5.9 \cdot 10^{-4}$ \\
\hline
\end{tabular}


The $d_{2}(m)$ listed in Table 3 are far too large to allow the completion of the class number $m$ problem using the computationally intensive sieve in $\S 6$ to investigate the range $d<d_{2}(m)$. Pushing the preceding arguments to their limit, it is possible to produce a $d_{2}^{*}(m)\left(<d_{2}(m)\right)$ such that if $d_{2}^{*}(m)<d<d_{3}(m)$, then $h(-d) \neq m$. Approximate values of $d_{2}^{*}(m)$ are given in Table 4 . Note, however, that for odd $m>9, d_{2}^{*}(m)$ is also far too large to allow one to complete the class number problem by using a computationally intensive sieve. Some type of further argument is necessary. In $\S 5.2$ and $\S 5.3$, the idea of partitioning minima is introduced, which allows us to reduce the range of admissible discriminants so that a computationally intensive sieve can be used. It turns out that our new arguments are powerful enough to rule out the range $d_{2}^{*}(m)<d<d_{2}(m)$, obviating the need to push the arguments of this subsection to their tedious limits.

Table 4. Lower bounds on $d$ obtained without partition estimates

\begin{tabular}{rcc}
\hline$m$ & $d_{2}^{*}(m)$ & $4(m-1)^{2 m}$ \\
\hline 5 & $4.5 \cdot 10^{12}$ & $4.2 \cdot 10^{6}$ \\
7 & $4.4 \cdot 10^{14}$ & $3.1 \cdot 10^{11}$ \\
9 & $2.2 \cdot 10^{18}$ & $7.2 \cdot 10^{16}$ \\
11 & $7.0 \cdot 10^{23}$ & $4.0 \cdot 10^{22}$ \\
13 & $8.7 \cdot 10^{29}$ & $4.6 \cdot 10^{28}$ \\
15 & $2.1 \cdot 10^{36}$ & $9.7 \cdot 10^{34}$ \\
17 & $8.4 \cdot 10^{42}$ & $3.5 \cdot 10^{41}$ \\
19 & $5.3 \cdot 10^{49}$ & $2.0 \cdot 10^{48}$ \\
21 & $5.0 \cdot 10^{56}$ & $1.8 \cdot 10^{55}$ \\
23 & $6.8 \cdot 10^{63}$ & $2.3 \cdot 10^{62}$ \\
\hline
\end{tabular}

The last column of Table 4 underscores the fact that the methods in this subsection do not suffice when $m>9$. This column arises from the fact that the denominator on the right-hand side of (5.9) must be positive. In other words, $d>4(m-1)^{2 m}$. Note that when $m>9$, the last column of Table 4 precludes the use of the computationally intensive sieve in $\S 6$, no matter how sharp we make the other estimates in this subsection.

5.2. The range $d_{1}(m) \leq d \leq d_{2}(m)$

Lemma 10. Let $t \in \mathbb{R}^{+}$. Assume that $m=h(-d)$ is odd and the partition $\mathcal{P}$ covers $M_{d}$ in the sense of Definition 2. Then

$$
\left|A_{d}(1 / 2+i t)\right| \geq 1-2 \sum_{a \in M_{d}^{*}} l_{a}(d)^{-1 / 2} .
$$

Furthermore, if the right-hand side of (5.21) is positive, then

$$
\left|\arg A_{d}(1 / 2+i t)\right| \leq \frac{2 t \sum_{a \in M_{d}^{*}} f\left(l_{a}(d)\right)}{1-2 \sum_{a \in M_{d}^{*}} l_{a}(d)^{-1 / 2}}
$$


where $f$ is the nonincreasing function defined by

$$
f(x)= \begin{cases}2 / e & \text { for } 0 \leq x \leq e^{2} \\ x^{-1 / 2} \log x & \text { for } x>e^{2}\end{cases}
$$

Proof. The proof is identical to the proof of Lemma 8 except that inequality (3.4) is replaced with the partition inequalities $l_{a}(d) \leq a \leq u_{a}(d)$.

Lemma 11. Let $t \in \mathbb{R}$ and $m=h(-d)>1$. Suppose that $m$ is odd and the partition $\mathcal{P}$ covers $M_{d}$ in the sense of Definition 2. If $k$ is an odd prime, then

$$
\begin{aligned}
\left|U_{d}(1 / 2+i t)\right| \leq & \frac{24 m(\pi \sqrt{3} k+2)}{d^{1 / 4}(\pi \sqrt{3})^{3 / 2} k^{2}} e^{-\pi \sqrt{3} k / 2} \\
& +\frac{16 \sqrt{k}(\pi \sqrt{d}+2 k)}{\pi^{3 / 2} d} e^{-\pi \sqrt{d} /(2 k)}+\frac{16}{d^{1 / 4}} \sum_{a \in M_{d}^{*}} F\left(\frac{\pi \sqrt{d}}{u_{a}(d) k}\right) .
\end{aligned}
$$

Proof. We use Lemma 9. In the outer sum of (5.15), $r=1$ or $r=k$. By Lemma 7, $F$ is decreasing, so we can bound the inner summands from above by using upper bounds on $a$. When $r=k$ we use the general upper bound $a \leq(d / 3)^{1 / 2}$ of (3.4). When $r=1$ and $a>1$ we use the partition inequality $a \leq u_{a}(d)$. Thus, we have

$$
\begin{aligned}
& \left|U_{d}(1 / 2+i t)\right| \\
& \quad \leq \frac{12 m}{d^{1 / 4} k^{1 / 2}} F(\pi \sqrt{3} k)+\frac{8}{d^{1 / 4}} F\left(\frac{\pi \sqrt{d}}{k}\right)+\frac{16}{d^{1 / 4}} \sum_{a \in M_{d}^{*}} F\left(\frac{\pi \sqrt{d}}{u_{a}(d) k}\right) .
\end{aligned}
$$

Using Lemma 6 with $N=0$ and $x=\pi \sqrt{3} k$ and the inequality $\log (1+x)<x$ (valid for $x>0$ ), we have

$$
|F(\pi \sqrt{3} k)| \leq \frac{2}{\sqrt{\pi} 3^{1 / 4} \sqrt{k}}\left(1+\frac{2}{\pi \sqrt{3} k}\right) e^{-\pi \sqrt{3} k / 2} .
$$

Similarly, for $x=\pi \sqrt{d} / k$, we obtain

$$
|F(\pi \sqrt{d} / k)| \leq \frac{2 \sqrt{k}}{\sqrt{\pi} d^{1 / 4}}\left(1+\frac{2 k}{\pi \sqrt{d}}\right) e^{-\pi \sqrt{d} /(2 k)} .
$$

Lemma 11 follows easily.

For the remainder of $\S 5.2$, we assume there exists a discriminant $-d$ satisfying the following properties:

(I) $h(-d)=m$, where $m \in\{5,7,9, \ldots, 23\}$ is fixed;

(II) $d_{1}(m) \leq d \leq d_{2}(m)$, where $d_{1}(m)$ is given in Table 5 near the end of this subsection and $d_{2}(m)$ is given in Table 3 . 
Our goal is to show that (5.7) is true for $d$ with a suitable choice of $k$ and $s_{0}$. In a manner similar to $\S 5.1$, we let $s_{0}=1 / 2+i t_{0}$ and use

$$
k=17923 \quad \text { and } \quad t_{0}=0.030985799 \text {. }
$$

Weinberger [31] has shown that the error in $t_{0}$ is less than $10^{-8}$ but we only use the first 5 significant digits. From (5.4)-(5.6) we then have

$$
\xi_{1}=0.01549, \quad \xi_{2}=0.2216, \quad \xi_{3}=57.1,
$$

where all approximations are accurate to the number of places shown.

As in $\S 3$, let $M_{d}$ denote the multiset of minima for $-d$. Turning to Appendix B, note that each entry in Table $\mathrm{B} \frac{m-3}{2}$ (e.g., when $m=15$, refer to Table B6) consists of $(m-1) / 2$ pairs of functions $\left(l_{a}(d), u_{a}(d)\right)$, each increasing in $d$, corresponding to the $(m-1) / 2$ elements in $M_{d}^{*}$. In other words, each entry of Table $\mathrm{B} \frac{m-3}{2}$ is a partition for $d$ according to Definition 1 in $\S 3$. Since $d \geq d_{1}(m) \geq 2^{m+2}$ from Table 5 , the arguments at the end of $\S 3$ show that there is some partition for $d$ in Table $\mathrm{B} \frac{m-3}{2}$ which covers $M_{d}$ in the sense of Definition 2 in $\S 3$. We henceforth denote this partition by $\mathcal{P}_{1}$.

Applying the first part of Lemma 10 with $t=t_{0}$ and $\mathcal{P}=\mathcal{P}_{1}$, we have

$$
\begin{aligned}
\left|A_{d}\left(s_{0}\right)\right| & \geq 1-2 \sum_{\left(l_{a}, u_{a}\right) \in \mathcal{P}_{1}} l_{a}(d)^{-1 / 2} \\
& \geq \min _{\mathcal{P}}\left(1-2 \sum_{\left(l_{a}, u_{a}\right) \in \mathcal{P}} l_{a}(d)^{-1 / 2}\right),
\end{aligned}
$$

where the minimum is henceforth understood to be over all partitions $\mathcal{P}$ for $d$ occurring in Table $\mathrm{B} \frac{m-3}{2}$. Since $l_{a}(d)$ is increasing in $d$, we replace $d$ with $d_{1}(m)$ in the lower bound of (5.23) giving the new lower bound

$$
\min _{\mathcal{P}}\left(1-2 \sum_{\left(l_{a}, u_{a}\right) \in \mathcal{P}} l_{a}\left(d_{1}(m)\right)^{-1 / 2}\right)>0
$$

with the positivity following from direct computation.

From $(5.23),(5.24)$, and Lemma 11 with $t=t_{0}, k=17923$, and $\mathcal{P}=\mathcal{P}_{1}$, we have

$$
\frac{\left|U_{d}\left(s_{0}\right)\right|}{\xi_{3}\left|A_{d}\left(s_{0}\right)\right|} \leq R_{1}\left(d, \mathcal{P}_{1}\right)
$$

where we define

$$
\begin{aligned}
R_{1}(d, \mathcal{P})= & \left\{\frac{24 m(\pi \sqrt{3} k+2)}{d^{1 / 4}(\pi \sqrt{3})^{3 / 2} k^{2}} e^{-\pi \sqrt{3} k / 2}+\frac{16(\pi \sqrt{d}+2 k)}{\pi^{3 / 2} d} e^{-\pi \sqrt{d} /(2 k)}\right. \\
& \left.+\frac{16}{d^{1 / 4}} \sum_{\left(l_{a}, u_{a}\right) \in \mathcal{P}} F\left(\frac{\pi \sqrt{d}}{u_{a}(d) k}\right)\right\} /\left\{\xi_{3}\left(1-2 \sum_{\left(l_{a}, u_{a}\right) \in \mathcal{P}} l_{a}(d)^{-1 / 2}\right)\right\} .
\end{aligned}
$$


Thus from (5.23) and (5.24) we have

$$
\frac{\left|U_{d}\left(s_{0}\right)\right|}{\xi_{3}\left|A_{d}\left(s_{0}\right)\right|} \leq \max _{\mathcal{P}} R_{1}(d, \mathcal{P})
$$

where the maximum is henceforth understood to be over all partitions $\mathcal{P}$ for $d$ occurring in Table $\mathrm{B} \frac{m-3}{2}$.

Note that for each $u_{a}$ occurring in each of the partitions for $d$ in Table $\mathrm{B} \frac{m-3}{2}, \sqrt{d} / u_{a}(d)$ is a nondecreasing function of $d$. It follows from Lemma 7 that $F\left(\pi \sqrt{d} /\left(u_{a}(d) k\right)\right)$ is a nonincreasing function of $d$. Therefore, the numerator of $R_{1}(d, \mathcal{P})$ is decreasing in $d$ for any partition $\mathcal{P}$ for $d$ appearing in Table $\mathrm{B} \frac{m-3}{2}$. Thus, with the aid of (5.24), we have

$$
\frac{\left|U_{d}\left(s_{0}\right)\right|}{\xi_{3}\left|A_{d}\left(s_{0}\right)\right|} \leq \max _{\mathcal{P}} R_{1}\left(d_{1}(m), \mathcal{P}\right)
$$

since $d \geq d_{1}(m)$. Upper bounds for $\max _{\mathcal{P}} R_{1}\left(d_{1}(m), \mathcal{P}\right)$ can be found in Table 5. In order to produce these approximations, we need estimates for the functions $F$ evaluated at small positive arguments. Such estimates are easily obtained by applying Lemma 6 with suitably large $N$.

Note that from (5.23) and (5.24) we know the right-hand side of (5.21) is positive for all partitions for $d$ appearing in Table $\mathrm{B} \frac{m-3}{2}$. Applying the second part of Lemma 10 with $t=t_{0}$ and $\mathcal{P}=\mathcal{P}_{1}$, we obtain

$$
\left|\arg A_{d}\left(s_{0}\right)\right| \leq \alpha_{1}\left(d, \mathcal{P}_{1}\right),
$$

where we define

$$
\alpha_{1}(d, \mathcal{P})=\frac{2 t_{0} \sum_{\left(l_{a}, u_{a}\right) \in \mathcal{P}} f\left(l_{a}(d)\right)}{1-2 \sum_{\left(l_{a}, u_{a}\right) \in \mathcal{P}} l_{a}(d)^{-1 / 2}} .
$$

It follows from the rightmost inequality in (5.23) and (5.24) that

$$
\left|\arg A_{d}\left(s_{0}\right)\right| \leq \max _{\mathcal{P}} \alpha_{1}(d, \mathcal{P}),
$$

where the maximum is over all partitions $\mathcal{P}$ for $d$ occurring in Table $\mathrm{B} \frac{m-3}{2}$.

Now $\alpha_{1}(d, \mathcal{P})$ is decreasing in $d$ for any fixed partition $\mathcal{P}$ for $d$ appearing in Table $\mathrm{B} \frac{m-3}{2}$. Hence,

$$
\left|\arg A_{d}\left(s_{0}\right)\right| \leq \max _{\mathcal{P}} \alpha_{1}\left(d_{1}(m), \mathcal{P}\right)
$$

since $d \geq d_{1}(m)$. Table 5 contains upper bounds for $\max _{\mathcal{P}} \alpha_{1}\left(d_{1}(m), \mathcal{P}\right)$.

Let $\beta_{1}(m)$ be defined by

$$
\beta_{1}(m)=\xi_{1} \log d_{1}(m)+\xi_{2}-\max _{\mathcal{P}} \alpha_{1}\left(d_{1}(m), \mathcal{P}\right)
$$

and $\gamma_{1}(m)$ be defined by

$$
\gamma_{1}(m)=\xi_{1} \log d_{2}(m)+\xi_{2}+\max _{\mathcal{P}} \alpha_{1}\left(10^{37}, \mathcal{P}\right) .
$$


Lower bounds for $\beta_{1}(m)$ and upper bounds for $\gamma_{1}(m)$ are given in Table 5 . Using Table 5, the fact that $d_{1}(m) \leq d$ and (5.27), we have

$$
0<\beta_{1}(m) \leq \xi_{1} \log d+\xi_{2}+\arg A_{d}\left(s_{0}\right) .
$$

In the other direction, we claim that

$$
\xi_{1} \log d+\xi_{2}+\arg A_{d}\left(s_{0}\right) \leq \gamma_{1}(m)<\pi .
$$

Note that from Tables 3 and 5 we have

$$
d_{1}(m)<10^{37}<d_{2}(m) .
$$

If $10^{37}<d$, our claim follows from the fact that $d \leq d_{2}(m),(5.26)$ coupled with the fact that $\alpha_{1}(d, \mathcal{P})$ is decreasing in $d$ for any fixed partition $\mathcal{P}$ for $d$ appearing in Table $\mathrm{B} \frac{m-3}{2}$, and Table 5 . When $d \leq 10^{37}$, note that by (5.27) we have

$$
\xi_{1} \log d+\xi_{2}+\arg A_{d}\left(s_{0}\right) \leq \xi_{1} \log \left(10^{37}\right)+\xi_{2}+\max _{\mathcal{P}} \alpha_{1}\left(d_{1}(m), \mathcal{P}\right) .
$$

With the aid of Table 5, direct calculation shows that

$$
\xi_{1} \log \left(10^{37}\right)+\xi_{2}+\max _{\mathcal{P}} \alpha_{1}\left(d_{1}(m), \mathcal{P}\right)<\gamma_{1}(m) .
$$

Thus our claim is also true when $d \leq 10^{37}$. Hence,

$$
\left|\sin \left(\xi_{1} \log d+\xi_{2}+\arg A_{d}\left(s_{0}\right)\right)\right| \geq \min \left\{\left|\sin \beta_{1}(m)\right|,\left|\sin \gamma_{1}(m)\right|\right\} .
$$

From the preceding inequality and Table 5 , we conclude that

$$
\left|\sin \left(\xi_{1} \log d+\xi_{2}+\arg A_{d}\left(s_{0}\right)\right)\right|>\max _{\mathcal{P}} R_{1}\left(d_{1}(m), \mathcal{P}\right) .
$$

In light of (5.25), (5.7) follows immediately. Since (5.3) is false, we conclude that $h(-d) \neq m$ for $m \in\{5,7, \ldots, 23\}$ and $d_{1}(m) \leq d \leq d_{2}(m)$.

Table 5. $h(-d) \neq m$ for $d_{1}(m) \leq d \leq d_{2}(m)$

\begin{tabular}{rcccccc}
\hline$m$ & $d_{1}(m)$ & $\max _{\mathcal{P}} R_{1}\left(d_{1}(m)\right)$ & $\max _{\mathcal{P}} \alpha_{1}\left(d_{1}(m), \mathcal{P}\right) \max _{\mathcal{P}} \alpha_{1}\left(10^{37}, \mathcal{P}\right)$ & $\beta_{1}(m)$ & $\gamma_{1}(m)$ \\
\hline 5 & $3.6 \cdot 10^{11}$ & 0.555 & 0.041 & $2.39 \cdot 10^{-4}$ & 0.592 & 2.542 \\
7 & $2.0 \cdot 10^{12}$ & 0.547 & 0.072 & $1.89 \cdot 10^{-3}$ & 0.588 & 2.543 \\
9 & $7.9 \cdot 10^{12}$ & 0.519 & 0.134 & $5.70 \cdot 10^{-3}$ & 0.547 & 2.583 \\
11 & $4.6 \cdot 10^{13}$ & 0.444 & 0.245 & $1.15 \cdot 10^{-2}$ & 0.463 & 2.660 \\
13 & $4.9 \cdot 10^{14}$ & 0.291 & 0.448 & $1.89 \cdot 10^{-2}$ & 0.297 & 2.846 \\
15 & $1.9 \cdot 10^{16}$ & 0.148 & 0.643 & $2.77 \cdot 10^{-2}$ & 0.158 & 2.961 \\
17 & $1.2 \cdot 10^{18}$ & 0.062 & 0.794 & $3.82 \cdot 10^{-2}$ & 0.072 & 3.079 \\
19 & $9.2 \cdot 10^{19}$ & 0.024 & 0.906 & $5.06 \cdot 10^{-2}$ & 0.027 & 3.091 \\
21 & $7.7 \cdot 10^{21}$ & 0.009 & 0.991 & $6.58 \cdot 10^{-2}$ & 0.010 & 3.107 \\
23 & $6.6 \cdot 10^{23}$ & 0.004 & 1.065 & $8.47 \cdot 10^{-2}$ & 0.005 & 3.125 \\
\hline
\end{tabular}

For $m \in\{5,7,9,11,13\}$, the $d_{1}(m)$ in Table 5 is small enough to allow the class number $m$ problem to be completed by the computationally intensive 
sieve of $\S 6$. However, for $m \in\{15,17,19,21,23\}$, we have to resort to further refinements in $\S 5.3$.

\subsection{The range $d_{0}(m) \leq d \leq d_{1}(m)$}

Lemma 12. Let $t \in \mathbb{R}^{+}$. Assume that $m=h(-d)$ is odd and the partition $\mathcal{P}$ covers $M_{d}$ in the sense of Definition 2. Let $p$ be the smallest nonprincipal minimum in $M_{d}$. If $l$ is a nonnegative integer such that the corresponding set $S_{l}=\left\{p, p^{2}, \ldots, p^{l}\right\} \subseteq M_{d}^{*}$ (where $S_{0}$ is understood to be the empty set), then

$$
\left|A_{d}(1 / 2+i t)\right| \geq\left|1-2 \frac{1-\lambda_{p}(t)^{l+1}}{1-\lambda_{p}(t)}\right|-2 \sum_{a \in M_{d}^{*}-S_{l}} l_{a}(d)^{-1 / 2},
$$

where $\lambda_{p}(t)=\chi(p) p^{-1 / 2-i t}$. Furthermore, if $\Re A_{d}(1 / 2+i \tau)>0$ for $0 \leq \tau \leq t$ and the right-hand side of (5.28) is positive, then

$$
\left|\arg A_{d}(1 / 2+i t)\right| \leq \frac{2 t\left(\log p\left|\sum_{j=1}^{l} j \lambda_{p}^{j}(t)\right|+\sum_{a} f\left(l_{a}(d)\right)\right)}{\left|1-2 \frac{1-\lambda_{p}^{l+1}(t)}{1-\lambda_{p}(t)}\right|-2 \sum_{a} l_{a}(d)^{-1 / 2}},
$$

where $f$ is as defined in Lemma 10 and the sums are over all $a \in M_{d}^{*}-S_{l}$.

Proof. The proof uses arguments similar to those in the proofs of Lemmas 8 and 10.

For the remainder of $\S 5.3$, we assume there exists a discriminant $-d$ satisfying the following properties:

(I) $h(-d)=m$, where $m \in\{9,11, \ldots, 23\}$ is fixed;

(II) $d_{0}(m) \leq d \leq d_{1}(m)$, where $d_{0}(m)$ is given in Table 6 near the end of this subsection and $d_{1}(m)$ is given in Table 5 .

Our goal is to show that (5.7) is true for $d$ with a suitable choice of $k$ and $s_{0}$. As in $\S 5.2$, let $s_{0}=1 / 2+i t_{0}$ and use

$$
k=17923 \text { and } t_{0}=0.030986 .
$$

From (5.4)-(5.6) we then have

$$
\xi_{1}=0.01549, \quad \xi_{2}=0.2216, \quad \xi_{3}=57.1,
$$

where all approximations are accurate to the number of places shown.

For each partition $\mathcal{P}$ for $d$ in Table $\mathrm{B} \frac{m-3}{2}$, define $\ell_{\mathcal{P}}$ to be the number of powers of the smallest minima in $M_{d}^{*}$ that can be shown to appear in $\mathcal{P}$ using the arguments of $\S 3$, if the number of such powers exceeds 2 . Otherwise, set $\ell_{\mathcal{P}}=0$. Denoting the smallest minimum in $M_{d}^{*}$ by $p$, let $S_{\ell_{\mathcal{P}}}=\left\{p, p^{2}, \ldots, p^{\ell_{\mathcal{P}}}\right\}$ if $\ell_{\mathcal{P}}>0$, and the empty set if $\ell_{\mathcal{P}}=0$. Finally, let $\mathcal{P}^{*}$ denote the set of $\left(l_{a}(d), u_{a}(d)\right)$ in $\mathcal{P}$ for which $a \notin S_{\ell_{\mathcal{P}}}$. 
Since $d \geq d_{0}(m)>2^{m+2}$ by Table 6 , the arguments at the end of $\S 3$ show there is some partition in Table $\mathrm{B} \frac{m-3}{2}$ which covers $M_{d}$ in the sense of Definition 2 in $\S 3$. We henceforth denote this partiton by $\mathcal{P}_{0}$. Applying the first part of Lemma 12 with $t=t_{0}, \mathcal{P}=\mathcal{P}_{0}, l=\ell_{\mathcal{P}_{0}}$, and letting $\lambda_{p}=\lambda_{p}\left(t_{0}\right)$, we have

$$
\begin{aligned}
\left|A_{d}\left(s_{0}\right)\right| & \geq\left|1-2 \frac{1-\lambda_{p}^{\ell_{\mathcal{P}_{0}}+1}}{1-\lambda_{p}}\right|-2 \sum_{\left(l_{a}, u_{a}\right) \in \mathcal{P}_{0}^{*}} l_{a}(d)^{-1 / 2} \\
& \geq \min _{\mathcal{P}}\left(\left|1-2 \frac{1-\lambda_{p}^{\ell_{\mathcal{P}}+1}}{1-\lambda_{p}}\right|-2 \sum_{\left(l_{a}, u_{a}\right) \in \mathcal{P}^{*}} l_{a}(d)^{-1 / 2}\right),
\end{aligned}
$$

where the minimum is henceforth understood to be over all partitions $\mathcal{P}$ for $d$ appearing in Table $\mathrm{B} \frac{m-3}{2}$. Let $\lambda_{q}=\chi(q) q^{-1 / 2-i t_{0}}$ and note that $l_{a}(d)$ and $u_{a}(d)$ are increasing in $d$. Note that we can find a further lower bound for the lower bound in (5.30) by evaluating the bound at all possible primes $p$. To this end, let

$$
\mathcal{Q}=\left\{q \text { prime }: l_{p}\left(d_{0}(m)\right) \leq q \leq u_{p}\left(d_{1}(m)\right),\left(l_{p}, u_{p}\right) \in \mathcal{P}\right\} .
$$

Now, a new lower bound for (5.30) is given by

$$
\min _{\mathcal{P}} \min _{q \in \mathcal{Q}}\left(\left|1-2 \frac{1-\lambda_{q}^{\ell_{\mathcal{P}}+1}}{1-\lambda_{q}}\right|-2 \sum_{\left(l_{a}, u_{a}\right) \in \mathcal{P}^{*}} l_{a}\left(d_{0}(m)\right)^{-1 / 2}\right)>0,
$$

with the positivity following from a direct computation. From (5.30), (5.31), and Lemma 11 with $t=t_{0}, k=17923$, and $\mathcal{P}=\mathcal{P}_{0}$, we have

$$
\frac{\left|U_{d}\left(s_{0}\right)\right|}{\xi_{3}\left|A_{d}\left(s_{0}\right)\right|} \leq R_{0}\left(d, \mathcal{P}_{0}\right)
$$

where we define

$$
\begin{aligned}
& R_{0}(d, \mathcal{P}) \\
& =\frac{\frac{24 m(\pi \sqrt{3} k+2)}{d^{1 / 4}(\pi \sqrt{3})^{3 / 2} k^{2}} e^{-\pi \sqrt{3} k / 2}+\frac{16(\pi \sqrt{d}+2 k)}{\pi^{3 / 2} d} e^{-\pi \sqrt{d} /(2 k)}+\frac{16}{d^{1 / 4}} \sum_{\mathcal{P}} F\left(\frac{\pi \sqrt{d}}{u_{a}(d) k}\right)}{\xi_{3}\left(\min _{q \in \mathcal{Q}}\left(\left|1-2 \frac{1-\lambda_{q}^{\ell_{\mathcal{P}}+1}}{1-\lambda_{q}}\right|-2 \sum_{\mathcal{P}^{*}} l_{a}\left(d_{0}(m)\right)^{-1 / 2}\right)\right)} .
\end{aligned}
$$

Thus from (5.30) and (5.31) we have

$$
\frac{\left|U_{d}\left(s_{0}\right)\right|}{\xi_{3}\left|A_{d}\left(s_{0}\right)\right|} \leq \max _{\mathcal{P}} R_{0}(d, \mathcal{P})
$$

where the maximum is henceforth understood to be over all partitions $\mathcal{P}$ for $d$ occurring in Table $\mathrm{B} \frac{m-3}{2}$. 
Note that for each $u_{a}$ occurring in each of the partitions for $d$ in Table $\mathrm{B} \frac{m-3}{2}, \sqrt{d} / u_{a}(d)$ is a nondecreasing function of $d$. Let $\mathcal{P}$ be any of the partitions for $d$ appearing in Table $\mathrm{B} \frac{m-3}{2}$. It follows from Lemma 7 that $F\left(\pi \sqrt{d} /\left(u_{a}(d) k\right)\right)$ is a nonincreasing function of $d$. Therefore, the numerator of $R_{0}(d, \mathcal{P})$ is decreasing in $d$ for any partition $\mathcal{P}$ for $d$ appearing in Table B $\frac{m-3}{2}$. Hence with the aid of (5.31) we have

$$
\frac{\left|U_{d}\left(s_{0}\right)\right|}{\xi_{3}\left|A_{d}\left(s_{0}\right)\right|} \leq \max _{\mathcal{P}} R_{0}\left(d_{0}(m), \mathcal{P}\right)
$$

Upper bounds for $\max _{\mathcal{P}} R_{0}\left(d_{0}(m), \mathcal{P}\right)$ can be found in Table 6 . In order to produce these approximations, we need estimates for the functions $F$ evaluated at small positive arguments. Such estimates are easily obtained by applying Lemma 6 with suitably large $N$.

Note that from (5.30) and (5.31) we know the right-hand side of (5.28) is positive for all partitions for $d$ appearing in Table $\mathrm{B} \frac{m-3}{2}$. Suppose that $0 \leq \tau \leq t_{0}$. If $\ell_{\mathcal{P}_{0}}=0$, then we have

$$
\begin{aligned}
\left|A_{d}(1 / 2+i \tau)-1\right| & \leq 2 \sum_{\left(l_{a}, u_{a}\right) \in \mathcal{P}_{0}} l_{a}(d)^{-1 / 2} \\
& \leq \max _{\mathcal{P}} 2 \sum_{\left(l_{a}, u_{a}\right) \in \mathcal{P}} l_{a}\left(d_{0}(m)\right)^{-1 / 2}<1,
\end{aligned}
$$

by a direct calculation. Hence, $\Re A_{d}(1 / 2+i \tau)>0$ when $\ell_{\mathcal{P}_{0}}=0$. On the other hand, suppose $\ell_{\mathcal{P}_{0}} \neq 0$. It follows from Table B $\frac{m-3}{2}$ and Table 5 that the smallest minimum $p \in M_{d}^{*}$ satisfies $p \leq\left(d_{1}(m) / 4\right)^{1 / 6} \leq 7406$. Hence, $0<2 t_{0} \log p<\pi$, and it is not difficult to see that

$$
\begin{aligned}
& \Re A_{d}(1 / 2+i \tau) \\
& \quad \geq 1-2 \sum_{j=1}^{\ell_{\mathcal{P}_{0}}} p^{-j / 2}+\frac{2+2 \cos \left(2 t_{0} \log p\right)}{p}-2 \sum_{\mathcal{P}_{0}^{*}} l_{a}\left(d_{0}(m)\right)^{-1 / 2} \\
& \quad \geq \min _{\mathcal{P}} \min _{q \in \mathcal{Q}}\left(1-2 \sum_{j=1}^{\ell_{\mathcal{P}}} q^{-j / 2}+\frac{2+2 \cos \left(2 t_{0} \log q\right)}{q}-2 \sum_{\mathcal{P}^{*}} l_{a}\left(d_{0}(m)\right)^{-1 / 2}\right) .
\end{aligned}
$$

It then follows by direct computation that $\Re A_{d}(1 / 2+i \tau)>0$ for $0 \leq \tau \leq t_{0}$. Hence, we may apply the second part of Lemma 12 with $t=t_{0}, \mathcal{P}=\mathcal{P}_{0}$, and $l=\ell_{\mathcal{P}_{0}}$ to obtain

$$
\left|\arg A_{d}\left(s_{0}\right)\right| \leq \frac{2 t_{0}\left(\log p\left|\sum_{j=1}^{\ell_{\mathcal{P}_{0}}} j \lambda_{p}^{j}\right|+\sum_{\left(l_{a}, u_{a}\right) \in \mathcal{P}_{0}^{*}} f\left(l_{a}(d)\right)\right)}{\left|1-2 \frac{1-\lambda_{p}^{\ell_{\mathcal{P}_{0}}}+1}{1-\lambda_{p}}\right|-2 \sum_{\left(l_{a}, u_{a}\right) \in \mathcal{P}_{0}^{*}} l_{a}(d)^{-1 / 2}}
$$


Hence, since $l_{a}(d)$ and $u_{a}(d)$ are increasing in $d$, we have

$$
\left|\arg A_{d}\left(s_{0}\right)\right| \leq \alpha_{0}\left(d, \mathcal{P}_{0}\right)
$$

where we define

$$
\alpha_{0}(d, \mathcal{P})=\max _{q \in \mathcal{Q}}\left(\frac{2 t_{0}\left(\log q\left|\sum_{j=1}^{\ell_{\mathcal{P}}} j \lambda_{q}^{j}\right|+\sum_{\left(l_{a}, u_{a}\right) \in \mathcal{P}^{*}} f\left(l_{a}(d)\right)\right)}{\left|1-2 \frac{1-\lambda_{q}^{\ell_{\mathcal{P}}+1}}{1-\lambda_{q}}\right|-2 \sum_{\left(l_{a}, u_{a}\right) \in \mathcal{P}^{*}} l_{a}(d)^{-1 / 2}}\right) .
$$

It follows from (5.30) and (5.31) that

$$
\left|\arg A_{d}\left(s_{0}\right)\right| \leq \max _{\mathcal{P}} \alpha_{0}(d, \mathcal{P})
$$

Since $f$ is nonincreasing and $l_{p}(d)$ is increasing in $d$, we know $\alpha_{0}(d, \mathcal{P})$ is decreasing in $d$ for any fixed partition $\mathcal{P}$ for $d$ appearing in Table $\mathrm{B} \frac{m-3}{2}$. Hence,

$$
\left|\arg A_{d}\left(s_{0}\right)\right| \leq \max _{\mathcal{P}} \alpha_{0}\left(d_{0}(m), \mathcal{P}\right)
$$

since $d \geq d_{0}(m)$. Table 6 contains upper bounds for $\max _{\mathcal{P}} \alpha_{0}\left(d_{0}(m), \mathcal{P}\right)$.

Let $\beta_{0}(m)$ be defined by

$$
\beta_{0}(m)=\xi_{1} \log d_{0}(m)+\xi_{2}-\max _{\mathcal{P}} \alpha_{0}\left(d_{0}(m), \mathcal{P}\right)
$$

and $\gamma_{0}(m)$ be defined by

$$
\gamma_{0}(m)=\xi_{1} \log d_{1}(m)+\xi_{2}+\max _{\mathcal{P}} \alpha_{0}\left(d_{0}(m), \mathcal{P}\right) .
$$

Lower bounds for $\beta_{0}(m)$ and upper bounds for $\gamma_{0}(m)$ are given in Table 6 . Using Table 6 , the fact that $d_{0}(m) \leq d$, and (5.33), we have

$$
0<\beta_{0}(m) \leq \xi_{1} \log d+\xi_{2}+\arg A_{d}\left(s_{0}\right) .
$$

Using the fact that $d \leq d_{1}(m),(5.33)$, and Table 6 , we have

$$
\xi_{1} \log d+\xi_{2}+\arg A_{d}\left(s_{0}\right) \leq \gamma_{0}(m)<\pi .
$$

Hence,

$$
\left|\sin \left(\xi_{1} \log d+\xi_{2}+\arg A_{d}\left(s_{0}\right)\right)\right| \geq \min \left\{\left|\sin \beta_{0}(m)\right|,\left|\sin \gamma_{0}(m)\right|\right\} .
$$

From the preceding inequality and Table 6 , we conclude that

$$
\left|\sin \left(\xi_{1} \log d+\xi_{2}+\arg A_{d}\left(s_{0}\right)\right)\right|>\max _{\mathcal{P}} R_{0}\left(d_{0}(m), \mathcal{P}\right) .
$$

In light of (5.32), (5.7) follows immediately. Since (5.3) is false, we conclude that $h(-d) \neq m$ for $m \in\{9,11, \ldots, 23\}$ and $d_{0}(m) \leq d \leq d_{1}(m)$. 
Table 6. $h(-d) \neq m$ for $d_{0}(m) \leq d \leq d_{1}(m)$

\begin{tabular}{rccccc}
\hline$m$ & $d_{0}(m)$ & $\max _{\mathcal{P}} R_{0}\left(d_{0}(m)\right)$ & $\max _{\mathcal{P}} \alpha_{0}\left(d_{0}(m), \mathcal{P}\right)$ & $\beta_{0}(m)$ & $\gamma_{0}(m)$ \\
\hline 9 & $6.4 \cdot 10^{12}$ & 0.553 & 0.089 & 0.589 & 0.771 \\
11 & $2.2 \cdot 10^{13}$ & 0.555 & 0.100 & 0.597 & 0.810 \\
13 & $4.2 \cdot 10^{13}$ & 0.556 & 0.116 & 0.591 & 0.862 \\
15 & $9.4 \cdot 10^{13}$ & 0.557 & 0.128 & 0.591 & 0.931 \\
17 & $1.9 \cdot 10^{14}$ & 0.556 & 0.138 & 0.592 & 1.005 \\
19 & $3.5 \cdot 10^{14}$ & 0.555 & 0.149 & 0.591 & 1.083 \\
21 & $6.5 \cdot 10^{14}$ & 0.542 & 0.173 & 0.576 & 1.176 \\
23 & $10.6 \cdot 10^{14}$ & 0.548 & 0.177 & 0.580 & 1.249 \\
\hline
\end{tabular}

A comparison of Tables 5 and 6 shows that the gap between $d_{0}(m)$ and $d_{1}(m)$ is increasing rapidly as $m$ increases from 9 to 23 .

6. The low range. In this section, we complete the proof of Theorem 1 , the statement of which appears in $\S 1$. Using the results of $\S 2$ and $\S 5$ (see Tables 5 and 6 in particular), it suffices to find all negative fundamental discriminants $-d$ with $h(-d) \in\{5,7, \ldots, 23\}$ such that $d \leq 1.1 \cdot 10^{15}$.

To this end, we first consider the small discriminants $d \leq 7.5 \cdot 10^{6}$ for which an exhaustive search is employed. For each $d$ in this range, we computed the class number by counting the number of reduced forms of discriminant $-d$. In other words, we searched for integers $a, b$, and $c$ with $0<a<(d / 3)^{1 / 2}$ and $c=\left(b^{2}-d\right) /(4 a)$ such that either $-a<b \leq a<c$ or $0 \leq b \leq a=c$. This straightforward approach required only 32 minutes on a Cray C90, rendering further optimization unnecessary. A complete listing of the negative fundamental discriminants with odd class numbers $m$ in the range $1 \leq m \leq 23$ that we found in this search is given in Appendix A. It is worth noting that Buell [6] had previously computed class numbers of imaginary quadratic number fields for $d \leq 4 \cdot 10^{6}$, and our results agree perfectly with his in this range. Furthermore, in recent unpublished work, Buell has independently verified our results up to $7.5 \cdot 10^{6}$, using our method of separating minima that was introduced in $\S 3$.

The largest value of $d$ found in the above search was $d=90787$. Thus, to complete the proof of Theorem 1 we need to show that there is no negative fundamental discriminant $-d$ with odd $h(-d) \leq 23$ in the range

$$
7.5 \cdot 10^{6} \leq d \leq 1.1 \cdot 10^{15}
$$

It is infeasible to directly check all $d$ in the range (6.1). Instead we used partition-type information in the following form.

LEMMA 13. If $d>8$ and $h(-d) \leq 23$ is odd, then

(i) $(-d \mid p) \neq 0$ for all primes $p<d$;

(ii) $(-d \mid p)=-1$ for all primes $p \leq(d / 4)^{1 / 23}$; 
(iii) $(-d \mid p)=-1$ for all primes $p \leq(d / 4)^{1 / 6}$ with at most one exception; and

(iv) $(-d \mid p)=-1$ for all primes $p \leq(d / 4)^{1 / 4}$ with at most two exceptions.

Proof. Item (i) follows directly from (2.1) and item (ii) follows from Lemmas 1 and 5 . If two odd primes $p, q \leq(d / 4)^{1 / 6}$ satisfy $(-d \mid p)=1$ and $(-d \mid q)=1$, then $p, p^{2}, p^{3}, q, q^{2}, q^{3}, p q, p^{2} q, p q^{2} \in M_{d}$, which implies $h(-d) \geq 25$ by Lemmas 1 and 4 . Thus, item (iii) is true. Lastly, if three primes $p, q, r \leq(d / 4)^{1 / 4}$ satisfy $(-d \mid p)=(-d \mid q)=(-d \mid r)=1$, then Lemmas 1 and 4 give $p, p^{2}, q, q^{2}, r, r^{2}, p q, p r, q r \in M_{d}$, which implies $h(-d) \geq 25$. This proves item (iv).

Now, we can build up a substantially smaller set (than (6.1)) of possible $d$ by using the Chinese Remainder Theorem on the residue requirements implicit in (i)-(iv) for a set of small primes. Consider an interval $d_{0} \leq d \leq d_{1}$. Let $p_{i}$ denote the $i$ th prime number and choose $k$ such that $m=8 \prod_{i=2}^{k} p_{i}>d_{1}$. Here, $m$ is the Chinese Remainder Theorem modulus when constructing integers $d$ based on the vector of Kronecker symbols $\left\langle\left(-d \mid p_{i}\right)\right\rangle_{1 \leq i \leq k}$. Let

$$
S_{k}(d)=\left\{\vec{\varepsilon} \in\{1,-1\}^{k}: \vec{\varepsilon}=\left\langle\left(-d \mid p_{i}\right)\right\rangle_{1 \leq i \leq k} \text { satisfies (i)-(iv) }\right\}
$$

and for each $\vec{\varepsilon} \in\{1,-1\}^{k}$ let

$$
D_{\vec{\varepsilon}}=\left\{0 \leq d<m:\left\langle\left(-d \mid p_{i}\right)\right\rangle_{1 \leq i \leq k}=\vec{\varepsilon}\right\} .
$$

To search all possible $d$ in $d_{0} \leq d \leq d_{1}$ we use the Chinese Remainder Theorem to construct $D_{\vec{\varepsilon}}$ for each $\vec{\varepsilon} \in S_{k}\left(d_{0}\right)$. The requirement that $(-d \mid p)=-1$ (or $(-d \mid p)=1)$ implies that $d$ is in one of $(p-1) / 2$ residue classes mod $p$ for odd primes $p$, and in one residue class $\bmod 8$ for $p=2$. Thus, $\left|D_{\vec{\varepsilon}}\right|=\prod_{i=2}^{k}\left(p_{i}-1\right) / 2 \approx d_{1} / 2^{k+3}$. Each $d \in \bigcup_{\vec{\varepsilon} \in S_{k}\left(d_{0}\right)} D_{\vec{\varepsilon}}$ is then checked using the necessary conditions (i)-(iv) for the primes $\left\{p_{k+1}, \ldots, p_{l}\right\}$ for $l$ suitably chosen whereby no $d$ satisfies the conditions. If $l$ exists, then there is no fundamental discriminant $-d$ with $d_{0} \leq d \leq d_{1}$ and $h(-d) \in\{1,3, \ldots, 23\}$. We use this approach on (6.1) by dividing it up into 3 subintervals corresponding to $k=10,12,13$.

First, consider $2.9 \cdot 10^{13} \leq d \leq 1.1 \cdot 10^{15}$. Take $k=13$ so that $m=$ $1217001054108840>1.1 \cdot 10^{\overline{15}}$. The bounds in (ii), (iii) and (iv) applied to $d_{0}=2.9 \cdot 10^{13}$ are 3,137 , and 1604 , respectively. Therefore, $(-d \mid 2)=$ $(-d \mid 3)=-1$ and at most one of the 11 primes in $\left\{p_{3}, \ldots, p_{13}\right\}$ satisfies $(-d \mid p)=1$. Hence, $\left|S_{13}\left(d_{0}\right)\right|=12$ resulting in at most $1.3 \cdot 10^{11}$ possible occurrences of $d \bmod m$. Using $l=56$, these were eliminated in 95 minutes on a Cray C90. As a check on the accuracy of the computer program, we printed out the last holdout, namely $d=123461955393043$. Note that $(-d \mid p)=-1$ for all primes $p \leq p_{56}=263$ except for $p=19,179$ and 263 . 
Next, consider $2.58 \cdot 10^{10} \leq d \leq 2.9 \cdot 10^{13}$. Take $k=12$ so that $m=$ $29682952539240>2.9 \cdot 10^{13}$. The bounds in (ii), (iii) and (iv) applied to $d_{0}=2.58 \cdot 10^{10}$ are 2,43 , and 283 , respectively. Therefore, $(-d \mid 2)=-1$ and at most one of the 11 primes in $\left\{p_{2}, \ldots, p_{12}\right\}$ satisfies $(-d \mid p)=1$. Hence, $\left|S_{12}\left(d_{0}\right)\right|=12$ resulting in at most $6.5 \cdot 10^{9}$ possible occurrences of $d \bmod m$. Using $l=51$, these were eliminated in 7.4 minutes on a Cray C90. Again as a check, note that for $d=7647157072003$ we have $(-d \mid p)=-1$ for all primes $p \leq p_{51}=233$ except for $p=43,67$ and 233 .

Lastly, consider $7.5 \cdot 10^{6} \leq d \leq 2.58 \cdot 10^{10}$. Take $k=10$ so that $m=$ $25878772920>2.58 \cdot 10^{10}$. The bounds in (ii), (iii) and (iv) applied to $d_{0}=7.5 \cdot 10^{6}$ are $1.8,11$, and 37 , respectively. In this case, $\left|S_{10}\left(d_{0}\right)\right|=46$ since among the first 10 primes there are 11 ways to have at most one exception and $5 \cdot 5+\left(\begin{array}{l}5 \\ 2\end{array}\right)$ ways to have exactly two exceptions. In order to eliminate the $9.2 \cdot 10^{7}$ constructed values of $d$ with primes greater than 37, we actually count the number of minima constructed with the primes satisfying $(-d \mid p)=1$. Note that Lemmas 2 and 3 imply that a prime in the range $37<p \leq 1369<\left(d_{0} / 4\right)^{1 / 2}$ satisfying $(-d \mid p)=1$ accounts for an additional 2 minima in $M_{d}$. When the count exceeds 23 , the value of $d$ can be eliminated.

\section{Appendix A. Negative fundamental discriminants for class numbers $1,3,5, \ldots, 23$}

Table A1. Values of $d$ with $h(-d)=1$

\begin{tabular}{lllllllll}
\hline 3 & 4 & 7 & 8 & 11 & 19 & 43 & 67 & 163 \\
\hline
\end{tabular}

Table A2. Values of $d$ with $h(-d)=3$

\begin{tabular}{rrrrrrrr}
\hline 23 & 31 & 59 & 83 & 107 & 139 & 211 & 283 \\
307 & 331 & 379 & 499 & 547 & 643 & 883 & 907 \\
\hline
\end{tabular}

Table A3. Values of $d$ with $h(-d)=5$

\begin{tabular}{rrrrrrrrr}
\hline 47 & 79 & 103 & 127 & 131 & 179 & 227 & 347 & 443 \\
523 & 571 & 619 & 683 & 691 & 739 & 787 & 947 & 1051 \\
1123 & 1723 & 1747 & 1867 & 2203 & 2347 & 2683 & & \\
\hline
\end{tabular}

Table A4. Values of $d$ with $h(-d)=7$

\begin{tabular}{rrrrrrrrr}
\hline 71 & 151 & 223 & 251 & 463 & 467 & 487 & 587 & 811 \\
827 & 859 & 1163 & 1171 & 1483 & 1523 & 1627 & 1787 & 1987 \\
2011 & 2083 & 2179 & 2251 & 2467 & 2707 & 3019 & 3067 & 3187 \\
3907 & 4603 & 5107 & 5923 & & & & & \\
\hline
\end{tabular}


Table A5. Values of $d$ with $h(-d)=9$

\begin{tabular}{rrrrrrrrr}
\hline 199 & 367 & 419 & 491 & 563 & 823 & 1087 & 1187 & 1291 \\
1423 & 1579 & 2003 & 2803 & 3163 & 3259 & 3307 & 3547 & 3643 \\
4027 & 4243 & 4363 & 4483 & 4723 & 4987 & 5443 & 6043 & 6427 \\
6763 & 6883 & 7723 & 8563 & 8803 & 9067 & 10627 & & \\
\hline
\end{tabular}

Table A6. Values of $d$ with $h(-d)=11$

\begin{tabular}{rrrrrrrr}
\hline 167 & 271 & 659 & 967 & 1283 & 1303 & 1307 & 1459 \\
1531 & 1699 & 2027 & 2267 & 2539 & 2731 & 2851 & 2971 \\
3203 & 3347 & 3499 & 3739 & 3931 & 4051 & 5179 & 5683 \\
6163 & 6547 & 7027 & 7507 & 7603 & 7867 & 8443 & 9283 \\
9403 & 9643 & 9787 & 10987 & 13003 & 13267 & 14107 & 14683 \\
15667 & & & & & & & \\
\hline
\end{tabular}

Table A7. Values of $d$ with $h(-d)=13$

\begin{tabular}{rrrrrrrr}
\hline 191 & 263 & 607 & 631 & 727 & 1019 & 1451 & 1499 \\
1667 & 1907 & 2131 & 2143 & 2371 & 2659 & 2963 & 3083 \\
3691 & 4003 & 4507 & 4643 & 5347 & 5419 & 5779 & 6619 \\
7243 & 7963 & 9547 & 9739 & 11467 & 11587 & 11827 & 11923 \\
12043 & 14347 & 15787 & 16963 & 20563 & & & \\
\hline
\end{tabular}

Table A8. Values of $d$ with $h(-d)=15$

\begin{tabular}{rrrrrrrr}
\hline 239 & 439 & 751 & 971 & 1259 & 1327 & 1427 & 1567 \\
1619 & 2243 & 2647 & 2699 & 2843 & 3331 & 3571 & 3803 \\
4099 & 4219 & 5003 & 5227 & 5323 & 5563 & 5827 & 5987 \\
6067 & 6091 & 6211 & 6571 & 7219 & 7459 & 7547 & 8467 \\
8707 & 8779 & 9043 & 9907 & 10243 & 10267 & 10459 & 10651 \\
10723 & 11083 & 11971 & 12163 & 12763 & 13147 & 13963 & 14323 \\
14827 & 14851 & 15187 & 15643 & 15907 & 16603 & 16843 & 17467 \\
17923 & 18043 & 18523 & 19387 & 19867 & 20707 & 22003 & 26203 \\
27883 & 29947 & 32323 & 34483 & & & & \\
\hline
\end{tabular}

Table A9. Values of $d$ with $h(-d)=17$

\begin{tabular}{rrrrrrrr}
\hline 383 & 991 & 1091 & 1571 & 1663 & 1783 & 2531 & 3323 \\
3947 & 4339 & 4447 & 4547 & 4651 & 5483 & 6203 & 6379 \\
6451 & 6827 & 6907 & 7883 & 8539 & 8731 & 9883 & 11251 \\
11443 & 12907 & 13627 & 14083 & 14779 & 14947 & 16699 & 17827 \\
18307 & 19963 & 21067 & 23563 & 24907 & 25243 & 26083 & 26107 \\
27763 & 31627 & 33427 & 36523 & 37123 & & & \\
\hline
\end{tabular}


Table A10. Values of $d$ with $h(-d)=19$

\begin{tabular}{rrrrrrrr}
\hline 311 & 359 & 919 & 1063 & 1543 & 1831 & 2099 & 2339 \\
2459 & 3343 & 3463 & 3467 & 3607 & 4019 & 4139 & 4327 \\
5059 & 5147 & 5527 & 5659 & 6803 & 8419 & 8923 & 8971 \\
9619 & 10891 & 11299 & 15091 & 15331 & 16363 & 16747 & 17011 \\
17299 & 17539 & 17683 & 19507 & 21187 & 21211 & 21283 & 23203 \\
24763 & 26227 & 27043 & 29803 & 31123 & 37507 & 38707 & \\
\hline
\end{tabular}

Table A11. Values of $d$ with $h(-d)=21$

\begin{tabular}{rrrrrrrr}
\hline 431 & 503 & 743 & 863 & 1931 & 2503 & 2579 & 2767 \\
2819 & 3011 & 3371 & 4283 & 4523 & 4691 & 5011 & 5647 \\
5851 & 5867 & 6323 & 6691 & 7907 & 8059 & 8123 & 8171 \\
8243 & 8387 & 8627 & 8747 & 9091 & 9187 & 9811 & 9859 \\
10067 & 10771 & 11731 & 12107 & 12547 & 13171 & 13291 & 13339 \\
13723 & 14419 & 14563 & 15427 & 16339 & 16987 & 17107 & 17707 \\
17971 & 18427 & 18979 & 19483 & 19531 & 19819 & 20947 & 21379 \\
22027 & 22483 & 22963 & 23227 & 23827 & 25603 & 26683 & 27427 \\
28387 & 28723 & 28867 & 31963 & 32803 & 34147 & 34963 & 35323 \\
36067 & 36187 & 39043 & 40483 & 44683 & 46027 & 49603 & 51283 \\
52627 & 55603 & 58963 & 59467 & 61483 & & & \\
\hline
\end{tabular}

Table A12. Values of $d$ with $h(-d)=23$

\begin{tabular}{rrrrrrrr}
\hline 647 & 1039 & 1103 & 1279 & 1447 & 1471 & 1811 & 1979 \\
2411 & 2671 & 3491 & 3539 & 3847 & 3923 & 4211 & 4783 \\
5387 & 5507 & 5531 & 6563 & 6659 & 6703 & 7043 & 9587 \\
9931 & 10867 & 10883 & 12203 & 12739 & 13099 & 13187 & 15307 \\
15451 & 16267 & 17203 & 17851 & 18379 & 20323 & 20443 & 20899 \\
21019 & 21163 & 22171 & 22531 & 24043 & 25147 & 25579 & 25939 \\
26251 & 26947 & 27283 & 28843 & 30187 & 31147 & 31267 & 32467 \\
34843 & 35107 & 37003 & 40627 & 40867 & 41203 & 42667 & 43003 \\
45427 & 45523 & 47947 & 90787 & & & & \\
\hline
\end{tabular}

Appendix B. Covering partitions for class numbers $5,7,9, \ldots, 23$. The following tables give a set of partitions covering all possible multisets $M_{d}$ under the assumption that $h(-d)=m$ for $m=5,7, \ldots, 23$. Let $p$ and $q$ denote the first and second smallest prime minima in $M_{d}$, respectively, and let $a$ denote a generic member of $M_{d}$. Let $v=d / 4$ and $w=d / 3$. The notation ( $n$ p.) implies that inequalities for the first $n$ powers of the prime are to be inferred. The notation $(n)$ simply means the inequality is to be listed a total of $n$ times. 
Table B1. Partitions for class number 5

\begin{tabular}{ll}
\hline 1 & $v^{1 / 5} \leq p \leq v^{1 / 4}(2 \mathrm{p})$. \\
2 & $v^{1 / 4} \leq p \leq v^{1 / 3}$ \\
3 & $v^{1 / 3} \leq a \leq w^{1 / 2}(2)$
\end{tabular}

Table B2. Partitions for class number 7

\begin{tabular}{lll}
\hline 1 & $v^{1 / 7} \leq p \leq v^{1 / 6} \quad(3 \mathrm{p})$. & \\
2 & $v^{1 / 6} \leq p \leq v^{1 / 4} \quad(2 \mathrm{p})$. & $v^{1 / 4} \leq a \leq w^{1 / 2}$ \\
3 & $v^{1 / 4} \leq p \leq v^{1 / 3}$ & $v^{1 / 4} \leq a \leq w^{1 / 2}(2)$ \\
4 & $v^{1 / 3} \leq a \leq w^{1 / 2}(3)$ & \\
\hline
\end{tabular}

Table B3. Partitions for class number 9

\begin{tabular}{lll}
\hline 1 & $v^{1 / 9} \leq p \leq v^{1 / 8} \quad(4 \mathrm{p})$. & \\
2 & $v^{1 / 8} \leq p \leq v^{1 / 6} \quad(3 \mathrm{p})$. & $v^{1 / 3} \leq a \leq w^{1 / 2}$ \\
3 & $v^{1 / 6} \leq p \leq v^{1 / 4} \quad(2 \mathrm{p})$. & $v^{1 / 4} \leq a \leq w^{1 / 2}(2)$ \\
4 & $v^{1 / 4} \leq p \leq v^{1 / 3}$ & $v^{1 / 4} \leq a \leq w^{1 / 2}$ \\
5 & $v^{1 / 3} \leq a \leq w^{1 / 2}$
\end{tabular}

Table B4. Partitions for class number 11

\begin{tabular}{llll}
\hline 1 & $v^{1 / 11} \leq p \leq v^{1 / 10}$ & $(5 \mathrm{p})$. & \\
2 & $v^{1 / 10} \leq p \leq v^{1 / 8}$ & $(4 \mathrm{p})$. & $v^{3 / 8} \leq a \leq w^{1 / 2}$ \\
3 & $v^{1 / 8} \leq p \leq v^{1 / 6}$ & $(3 \mathrm{p})$. & $v^{1 / 3} \leq a \leq w^{1 / 2}$ \\
4 & $v^{1 / 6} \leq p \leq v^{1 / 4}$ & $(2 \mathrm{p})$. & $v^{1 / 4} \leq a \leq w^{1 / 2}$ \\
5 & $v^{1 / 4} \leq p \leq v^{1 / 3}$ & & $v^{1 / 4} \leq a \leq w^{1 / 2}$ \\
6 & $v^{1 / 3} \leq a \leq w^{1 / 2}$ & $(5)$ \\
\hline
\end{tabular}

Table B5. Partitions for class number 13

\begin{tabular}{|c|c|c|c|c|}
\hline 1 & $v^{1 / 13} \leq p \leq v^{1 / 12}$ & (6p.) & & \\
\hline 2 & $v^{1 / 12} \leq p \leq v^{1 / 10}$ & (5p.) & $v^{2 / 5} \leq a \leq w^{1 / 2}$ & \\
\hline 3 & $v^{1 / 10} \leq p \leq v^{1 / 8}$ & $(4 \mathrm{p})$. & $v^{3 / 8} \leq a \leq w^{1 / 2}(2)$ & \\
\hline 4 & $v^{1 / 8} \leq p \leq v^{1 / 6}$ & $(3 \mathrm{p})$. & $v^{1 / 4} \leq q \leq v^{1 / 3}$ & $v^{3 / 8} \leq p q \leq v^{1 / 2}$ \\
\hline 5 & $v^{1 / 8} \leq p \leq v^{1 / 6}$ & (3p.) & $v^{1 / 3} \leq a \leq w^{1 / 2}$ & \\
\hline 6 & $v^{1 / 6} \leq p \leq v^{1 / 4}$ & (2p.) & $v^{1 / 6} \leq q \leq v^{1 / 4}$ & $v^{1 / 3} \leq p q \leq v^{1 / 2}$ \\
\hline 7 & $v^{1 / 6} \leq p \leq v^{1 / 4}$ & $(2 \mathrm{p})$. & $v^{1 / 4} \leq q \leq v^{1 / 3}$ & $v^{1 / 4} \leq a \leq w^{1 / 2}$ \\
\hline 8 & $v^{1 / 6} \leq p \leq v^{1 / 4}$ & $(2 \mathrm{p})$. & $v^{1 / 3} \leq a \leq w^{1 / 2}$ & \\
\hline 9 & $v^{1 / 4} \leq p \leq v^{1 / 3}$ & & $v^{1 / 4} \leq a \leq w^{1 / 2}$ & \\
\hline 10 & $v^{1 / 3} \leq a \leq w^{1 / 2}$ & (6) & & \\
\hline
\end{tabular}


Table B6. Partitions for class number 15

\begin{tabular}{|c|c|c|c|c|}
\hline 1 & $v^{1 / 15} \leq p \leq v^{1 / 14}$ & (7p.) & & \\
\hline 2 & $v^{1 / 14} \leq p \leq v^{1 / 12}$ & (6p.) & $v^{5 / 12} \leq a \leq w^{1 / 2}$ & \\
\hline 3 & $v^{1 / 12} \leq p \leq v^{1 / 10}$ & (5p.) & $v^{2 / 5} \leq a \leq w^{1 / 2}(2)$ & \\
\hline 4 & $v^{1 / 10} \leq p \leq v^{1 / 8}$ & $(4 p)$. & $v^{1 / 4} \leq a \leq w^{1 / 2}$ & \\
\hline 5 & $v^{1 / 8} \leq p \leq v^{1 / 6}$ & (3p.) & $v^{1 / 6} \leq q \leq v^{1 / 4}$ & $v^{7 / 24} \leq p q \leq v^{5 / 12}$ \\
\hline 6 & $v^{1 / 8} \leq p \leq v^{1 / 6}$ & (3p.) & $v^{1 / 4} \leq q \leq v^{1 / 3}$ & $\begin{array}{l}v^{3 / 8} \leq p q \leq v^{1 / 2} \\
v^{1 / 3} \leq a \leq w^{1 / 2}\end{array}$ \\
\hline 7 & $v^{1 / 8} \leq p \leq v^{1 / 6}$ & (3p.) & $v^{1 / 3} \leq a \leq w^{1 / 2}$ & \\
\hline 8 & $v^{1 / 6} \leq p \leq v^{1 / 4}$ & (2p.) & $v^{1 / 6} \leq q \leq v^{1 / 4}$ & $\begin{array}{l}v^{1 / 3} \leq p q \leq v^{1 / 2} \\
v^{1 / 4} \leq a \leq w^{1 / 2}\end{array}$ \\
\hline 9 & $v^{1 / 6} \leq p \leq v^{1 / 4}$ & (2p.) & $v^{1 / 4} \leq q \leq v^{1 / 3}$ & $v^{1 / 4} \leq a \leq w^{1 / 2}$ \\
\hline 10 & $v^{1 / 6} \leq p \leq v^{1 / 4}$ & (2p.) & $v^{1 / 3} \leq a \leq w^{1 / 2}$ & \\
\hline 11 & $v^{1 / 4} \leq p \leq v^{1 / 3}$ & & $v^{1 / 4} \leq a \leq w^{1 / 2}(6)$ & \\
\hline 12 & $v^{1 / 3} \leq a \leq w^{1 / 2}$ & (7) & & \\
\hline
\end{tabular}

Table B7. Partitions for class number 17

\begin{tabular}{|c|c|c|c|c|c|c|}
\hline 1 & $v^{1 / 17} \leq p \leq v^{1 / 16}$ & $(8 p)$. & & & & \\
\hline 2 & $v^{1 / 16} \leq p \leq v^{1 / 14}$ & $(7 \mathrm{p})$. & $v^{3 / 7} \leq a \leq w^{1 / 2}$ & & & \\
\hline 3 & $v^{1 / 14} \leq p \leq v^{1 / 12}$ & $(6 \mathrm{p})$. & $v^{5 / 12} \leq a \leq w^{1 / 2}$ & (2) & & \\
\hline 4 & $v^{1 / 12} \leq p \leq v^{1 / 10}$ & $(5 \mathrm{p})$. & $v^{3 / 10} \leq a \leq w^{1 / 2}$ & (3) & & \\
\hline 5 & $v^{1 / 10} \leq p \leq v^{1 / 8}$ & $(4 p)$. & $v^{1 / 4} \leq q \leq v^{3 / 8}$ & & $\begin{array}{l}v^{7 / 20} \leq p q \leq v^{1 / 2} \\
v^{3 / 8} \leq a \leq w^{1 / 2}\end{array}$ & (2) \\
\hline 6 & $v^{1 / 10} \leq p \leq v^{1 / 8}$ & $(4 \mathrm{p})$. & $v^{3 / 8} \leq a \leq w^{1 / 2}$ & (4) & & \\
\hline 7 & $v^{1 / 8} \leq p \leq v^{1 / 6}$ & $(3 \mathrm{p})$. & $v^{1 / 6} \leq q \leq v^{1 / 4}$ & (2p.) & $\begin{array}{l}v^{7 / 24} \leq p q \leq v^{5 / 12} \\
v^{1 / 3} \leq a \leq w^{1 / 2}\end{array}$ & (2) \\
\hline 8 & $v^{1 / 8} \leq p \leq v^{1 / 6}$ & $(3 \mathrm{p})$. & $v^{1 / 4} \leq q \leq v^{1 / 3}$ & & $\begin{array}{l}v^{3 / 8} \leq p q \leq v^{1 / 2} \\
v^{1 / 3} \leq a \leq w^{1 / 2}\end{array}$ & $\begin{array}{l}(2) \\
(2)\end{array}$ \\
\hline 9 & $v^{1 / 8} \leq p \leq v^{1 / 6}$ & $(3 \mathrm{p})$. & $v^{1 / 3} \leq a \leq w^{1 / 2}$ & (5) & & \\
\hline 10 & $v^{1 / 6} \leq p \leq v^{1 / 4}$ & (2p.) & $v^{1 / 6} \leq q \leq v^{1 / 4}$ & $(2 \mathrm{p})$. & $\begin{array}{l}v^{1 / 3} \leq p q \leq v^{1 / 2} \\
v^{1 / 4} \leq a \leq w^{1 / 2}\end{array}$ & $\begin{array}{l}(2) \\
(2)\end{array}$ \\
\hline 11 & $v^{1 / 6} \leq p \leq v^{1 / 4}$ & $(2 \mathrm{p})$. & $v^{1 / 4} \leq q \leq v^{1 / 3}$ & & $v^{1 / 4} \leq a \leq w^{1 / 2}$ & (5) \\
\hline 12 & $v^{1 / 6} \leq p \leq v^{1 / 4}$ & (2p.) & $v^{1 / 3} \leq a \leq w^{1 / 2}$ & (6) & & \\
\hline 13 & $v^{1 / 4} \leq p \leq v^{1 / 3}$ & & $v^{1 / 4} \leq a \leq w^{1 / 2}$ & (7) & & \\
\hline 14 & $v^{1 / 3} \leq a \leq w^{1 / 2}$ & (8) & & & & \\
\hline
\end{tabular}


Table B8. Partitions for class number 19

\begin{tabular}{|c|c|c|c|c|c|c|}
\hline 1 & $v^{1 / 19} \leq p \leq v^{1 / 18}$ & $(9 \mathrm{p})$. & & & & \\
\hline 2 & $v^{1 / 18} \leq p \leq v^{1 / 16}$ & $(8 p)$. & $v^{7 / 16} \leq a \leq w^{1 / 2}$ & & & \\
\hline 3 & $v^{1 / 16} \leq p \leq v^{1 / 14}$ & $4(7 p)$. & $v^{3 / 7} \leq a \leq w^{1 / 2}$ & $(2)$ & & \\
\hline 4 & $v^{1 / 14} \leq p \leq v^{1 / 12}$ & $(6 \mathrm{p})$. & $v^{1 / 3} \leq a \leq w^{1 / 2}$ & (3) & & \\
\hline 5 & $v^{1 / 12} \leq p \leq v^{1 / 10}$ & $(5 p)$. & $v^{3 / 10} \leq a \leq w^{1 / 2}$ & (4) & & \\
\hline 6 & $v^{1 / 10} \leq p \leq v^{1 / 8}$ & $(4 p)$. & $v^{1 / 4} \leq q \leq v^{3 / 8}$ & & $v^{7 / 20} \leq p q \leq v^{1 / 2}$ & (2) \\
\hline 7 & $v^{1 / 10} \leq p \leq v^{1 / 8}$ & $(4 \mathrm{p})$. & $v^{3 / 8} \leq a \leq w^{1 / 2}$ & $(5)$ & $v^{3 / 8} \leq a \leq w^{1 / 2}$ & (2) \\
\hline 8 & $v^{1 / 8} \leq p \leq v^{1 / 6}$ & (3p.) & $v^{1 / 6} \leq q \leq v^{1 / 4}$ & $(2 \mathrm{p})$. & $\begin{array}{l}v^{7 / 24} \leq p q \leq v^{5 / 12} \\
v^{1 / 3}<a<w^{1 / 2}\end{array}$ & $\begin{array}{l}(2) \\
(2)\end{array}$ \\
\hline 9 & $v^{1 / 8} \leq p \leq v^{1 / 6}$ & (3p.) & $v^{1 / 4} \leq q \leq v^{1 / 3}$ & & $\begin{array}{l}v^{3 / 8} \leq p q \leq v^{1 / 2} \\
v^{1 / 4} \leq a \leq w^{1 / 2}\end{array}$ & $\begin{array}{l}(2) \\
(3)\end{array}$ \\
\hline 10 & $v^{1 / 8} \leq p \leq v^{1 / 6}$ & (3p.) & $v^{1 / 3} \leq a \leq w^{1 / 2}$ & $(6)$ & & \\
\hline 11 & $v^{1 / 6} \leq p \leq v^{1 / 4}$ & $(2 \mathrm{p})$. & $v^{1 / 6} \leq q \leq v^{1 / 4}$ & $(2 \mathrm{p})$. & $\begin{array}{l}v^{1 / 3} \leq p q \leq v^{1 / 2} \\
v^{1 / 4} \leq a \leq w^{1 / 2}\end{array}$ & $\begin{array}{l}(2) \\
(3)\end{array}$ \\
\hline 12 & $v^{1 / 6} \leq p \leq v^{1 / 4}$ & (2p.) & $v^{1 / 4} \leq q \leq v^{1 / 3}$ & & $v^{1 / 4} \leq a \leq w^{1 / 2}$ & (6) \\
\hline 13 & $v^{1 / 6} \leq p \leq v^{1 / 4}$ & $(2 \mathrm{p})$. & $v^{1 / 3} \leq a \leq w^{1 / 2}$ & $(7)$ & & \\
\hline 14 & $v^{1 / 4} \leq p \leq v^{1 / 3}$ & & $v^{1 / 4} \leq a \leq w^{1 / 2}$ & (8) & & \\
\hline 15 & $v^{1 / 3} \leq a \leq w^{1 / 2}$ & (9) & & & & \\
\hline
\end{tabular}

Table B9. Partitions for class number 21

\begin{tabular}{|c|c|c|c|c|}
\hline 1 & $v^{1 / 21} \leq p \leq v^{1 / 20}$ & & & \\
\hline 2 & $v^{1 / 20} \leq p \leq v^{1 / 18}(9 \mathrm{p})$. & $v^{4 / 9} \leq a \leq w^{1 / 2}$ & & \\
\hline 3 & $v^{1 / 18} \leq p \leq v^{1 / 16}(8 \mathrm{p})$. & $v^{7 / 16} \leq a \leq w^{1 / 2}(2)$ & & \\
\hline 4 & $v^{1 / 16} \leq p \leq v^{1 / 14}(7 \mathrm{p})$. & $v^{5 / 14} \leq a \leq w^{1 / 2}$ & & \\
\hline 5 & $v^{1 / 14} \leq p \leq v^{1 / 12}(6 \mathrm{p})$. & $v^{1 / 3} \leq a \leq w^{1 / 2}$ & & \\
\hline 6 & $v^{1 / 12} \leq p \leq v^{1 / 10}(5 \mathrm{p})$. & $v^{1 / 4} \leq q \leq v^{3 / 10}$ & $\begin{array}{l}v^{1 / 3} \leq p q \leq v^{2 / 5} \\
v^{5 / 12} \leq p^{2} q \leq v^{1 / 2}\end{array}$ & $\begin{array}{l}\text { 2) } \\
\text { 2) }\end{array}$ \\
\hline 7 & $v^{1 / 12} \leq p \leq v^{1 / 10}$ & $v^{3 / 10} \leq a \leq w^{1 / 2}(5)$ & & \\
\hline 8 & $v^{1 / 10} \leq p \leq v^{1 / 8}$ & $v^{3 / 16} \leq q \leq v^{1 / 4}$ & $\begin{aligned} v^{23 / 80} & \leq p q \leq v^{3 / 8} \\
v^{31 / 80} & \leq p^{2} q \leq v^{1 / 2}\end{aligned}$ & $\begin{array}{l}\text { 2) } \\
\text { 2) }\end{array}$ \\
\hline 9 & $v^{1 / 10} \leq p \leq v^{1 / 8}$ & $v^{1 / 4} \leq q \leq v^{3 / 8}$ & $\begin{array}{l}v^{7 / 20} \leq p q \leq v^{1 / 2} \\
v^{1 / 4} \leq a \leq w^{1 / 2}\end{array}$ & $\begin{array}{l}\text { 2) } \\
\text { 3) }\end{array}$ \\
\hline 10 & $v^{1 / 10} \leq p \leq v^{1 / 8}$ & $v^{3 / 8} \leq a \leq w^{1 / 2}$ & & \\
\hline
\end{tabular}


Table B9 (cont.)

\begin{tabular}{|c|c|c|c|c|}
\hline 11 & $v^{1 / 8} \leq p \leq v^{1 / 6}$ & $(3 p)$. & $v^{1 / 6} \leq q \leq v^{1 / 4}$ & $\begin{array}{l}v^{7 / 24} \leq p q \leq v^{5 / 12} \\
v^{1 / 4} \leq a \leq w^{1 / 2}\end{array}$ \\
\hline 12 & $v^{1 / 8} \leq p \leq v^{1 / 6}$ & $(3 p)$. & $v^{1 / 4} \leq q \leq v^{1 / 3}$ & $\begin{array}{l}v^{3 / 8} \leq p q \leq v^{1 / 2} \\
v^{1 / 4} \leq a \leq w^{1 / 2}\end{array}$ \\
\hline 13 & $v^{1 / 8} \leq p \leq v^{1 / 6}$ & $(3 p)$. & $v^{1 / 3} \leq a \leq w^{1 / 2}(7)$ & \\
\hline 14 & $v^{1 / 6} \leq p \leq v^{1 / 4}$ & $(2 \mathrm{p})$. & $v^{1 / 6} \leq q \leq v^{1 / 4}$ & $\begin{aligned} v^{1 / 3} & \leq p q \leq v^{1 / 2} \\
v^{1 / 4} & \leq a \leq w^{1 / 2}\end{aligned}$ \\
\hline 15 & $v^{1 / 6} \leq p \leq v^{1 / 4}$ & $(2 \mathrm{p})$. & $v^{1 / 4} \leq q \leq v^{1 / 3}$ & $v^{1 / 4} \leq a \leq w^{1 / 2}$ \\
\hline 16 & $v^{1 / 6} \leq p \leq v^{1 / 4}$ & $(2 \mathrm{p})$. & $v^{1 / 3} \leq a \leq w^{1 / 2}$ & \\
\hline 17 & $v^{1 / 4} \leq p \leq v^{1 / 3}$ & & $v^{1 / 4} \leq a \leq w^{1 / 2}(9)$ & \\
\hline 18 & $v^{1 / 3} \leq a \leq w^{1 / 2}$ & (10) & & \\
\hline
\end{tabular}

Table B10. Partitions for class number 23

\begin{tabular}{|c|c|c|c|c|c|}
\hline 1 & $v^{1 / 23} \leq p \leq v^{1 / 22}$ & & & & \\
\hline 2 & $v^{1 / 22} \leq p \leq v^{1 / 20}$ & $v^{9 / 20} \leq a \leq w^{1 / 2}$ & & & \\
\hline 3 & $v^{1 / 20} \leq p \leq v^{1 / 18}(9 \mathrm{p})$. & $v^{4 / 9} \leq a \leq w^{1 / 2}$ & (2) & & \\
\hline 4 & $v^{1 / 18} \leq p \leq v^{1 / 16}(8 \mathrm{p})$. & $v^{3 / 8} \leq a \leq w^{1 / 2}$ & (3) & & \\
\hline 5 & $v^{1 / 16} \leq p \leq v^{1 / 14}(7 \mathrm{p})$. & $v^{5 / 14} \leq a \leq w^{1 / 2}$ & (4) & & \\
\hline 6 & $v^{1 / 14} \leq p \leq v^{1 / 12}(6 \mathrm{p})$. & $v^{1 / 4} \leq q \leq v^{1 / 3}$ & & $\begin{array}{l}v^{9 / 28} \leq p q \leq v^{5 / 12} \\
v^{11 / 28} \leq p^{2} q \leq v^{1 / 2}\end{array}$ & $\begin{array}{l}(2) \\
(2)\end{array}$ \\
\hline 7 & $v^{1 / 14} \leq p \leq v^{1 / 12}(6 \mathrm{p})$. & $v^{1 / 3} \leq a \leq w^{1 / 2}$ & $(5)$ & & \\
\hline 8 & $v^{1 / 12} \leq p \leq v^{1 / 10}$ & $\begin{array}{l}v^{1 / 5} \leq q \leq v^{3 / 10} \\
v^{11 / 30} \leq p^{2} q \leq v^{1 / 2}\end{array}$ & $(2)$ & $\begin{array}{l}v^{17 / 60} \leq p q \leq v^{2 / 5} \\
v^{2 / 5} \leq a \leq w^{1 / 2}\end{array}$ & \\
\hline 9 & $v^{1 / 12} \leq p \leq v^{1 / 10}(5 \mathrm{p})$. & $v^{3 / 10} \leq a \leq w^{1 / 2}$ & $(6)$ & & \\
\hline 10 & $v^{1 / 10} \leq p \leq v^{1 / 8}$ & $\begin{array}{l}v^{3 / 16} \leq q \leq v^{1 / 4} \\
v^{31 / 80} \leq p^{2} q \leq v^{1 / 2}\end{array}$ & $\begin{array}{l}(2 \mathrm{p} .) \\
(2)\end{array}$ & $\begin{array}{l}v^{23 / 80} \leq p q \leq v^{3 / 8} \\
v^{3 / 8} \leq a \leq w^{1 / 2}\end{array}$ & (2) \\
\hline 11 & $v^{1 / 10} \leq p \leq v^{1 / 8}$ & $v^{1 / 4} \leq q \leq v^{3 / 8}$ & & $\begin{array}{l}v^{7 / 20} \leq p q \leq v^{1 / 2} \\
v^{1 / 4} \leq a \leq w^{1 / 2}\end{array}$ & $\begin{array}{l}(2) \\
(4)\end{array}$ \\
\hline 12 & $v^{1 / 10} \leq p \leq v^{1 / 8}$ & $v^{3 / 8} \leq a \leq w^{1 / 2}$ & (7) & & \\
\hline 13 & $v^{1 / 8} \leq p \leq v^{1 / 6}$ & $v^{1 / 6} \leq q \leq v^{1 / 4}$ & $(2 \mathrm{p})$. & $\begin{array}{l}v^{7 / 24} \leq p q \leq v^{5 / 12} \\
v^{1 / 4} \leq a \leq w^{1 / 2}\end{array}$ & $\begin{array}{l}(2) \\
(4)\end{array}$ \\
\hline 14 & $v^{1 / 8} \leq p \leq v^{1 / 6}$ & $v^{1 / 4} \leq q \leq v^{1 / 3}$ & & $\begin{array}{l}v^{3 / 8} \leq p q \leq v^{1 / 2} \\
v^{1 / 4} \leq a \leq w^{1 / 2}\end{array}$ & $\begin{array}{l}(2) \\
(5)\end{array}$ \\
\hline
\end{tabular}


Table B10 (cont.)

\begin{tabular}{|c|c|c|c|}
\hline 15 & $v^{1 / 8} \leq p \leq v^{1 / 6}$ & $v^{1 / 3} \leq a \leq w^{1 / 2}$ & \\
\hline 16 & $v^{1 / 6} \leq p \leq v^{1 / 4}$ & $v^{1 / 6} \leq q \leq v^{1 / 4}$ & $\begin{array}{l}v^{1 / 3} \leq p q \leq v^{1 / 2} \\
v^{1 / 4} \leq a \leq w^{1 / 2}\end{array}$ \\
\hline 17 & $v^{1 / 6} \leq p \leq v^{1 / 4}$ & $v^{1 / 4} \leq q \leq v^{1 / 3}$ & $v^{1 / 4} \leq a \leq w^{1 / 2}$ \\
\hline 18 & $v^{1 / 6} \leq p \leq v^{1 / 4}$ & $v^{1 / 3} \leq a \leq w^{1 / 2}$ & \\
\hline 19 & $v^{1 / 4} \leq p \leq v^{1 / 3}$ & $v^{1 / 4} \leq a \leq w^{1 / 2}(10)$ & \\
\hline 20 & $v^{1 / 3} \leq a \leq w^{1 / 2}$ & & \\
\hline
\end{tabular}

Note added in proof. C. Wagner has independently solved the class number 5 and 7 problems (and the class number 6 problem as well) in a paper: Class number 5, 6 and 7 , Math. Comp. 65 (1996), 785-800.

Acknowledgements. The authors would like to express their gratitude to the referee for suggesting several improvements. All of our numerical computations and explorations were done with the aid of Mathematica on a SPARCstation, with the exception of the C code written for the Cray C90 discussed in $\S 6$.

\section{References}

[1] S. Arno, The imaginary quadratic fields of class number 4, Acta Arith. 60 (1992), 321-334.

[2] A. Baker, Linear forms in the logarithms of algebraic numbers. I, Mathematika 13 (1966), 204-216.

[3] -, Imaginary quadratic fields with class number 2, Ann. of Math. 94 (1971), 139152.

[4] -, Transcendental Number Theory, Cambridge Univ. Press, New York, 1975.

[5] B. J. Birch and H. P. F. Swinnerton-Dyer, Notes on elliptic curves. II, J. Reine Angew. Math. 218 (1965), 79-108.

[6] D. A. Buell, Class groups of quadratic fields. II, Math. Comp. 48 (1987), 85-93.

[7] H. Daven port, Multiplicative Number Theory, 2nd ed., Grad. Texts in Math. 74, Springer, New York, 1980.

[8] M. Deuring, Imaginäre quadratische Zahlkörper mit der Klassenzahl Eins, Invent. Math. 5 (1968), 169-179.

[9] C. F. Gauss, Disquisitiones Arithmeticae, Yale Univ. Press, 1966.

[10] D. M. Goldfeld, The class number of quadratic fields and the conjectures of Birch and Swinnerton-Dyer, Ann. Scuola Norm. Sup. Pisa 3 (1976), 623-663.

[11] B. Gross et D. Zagier, Points de Heegner et derivées de fonctions L, C. R. Acad. Sci. Paris 297 (1983), 85-87.

[12] G. H. Hardy and E. M. Wright, An Introduction to the Theory of Numbers, 4th ed., Oxford Univ. Press, London, 1968. 
[13] K. Heegner, Diophantische Analysis und Modulfunktionen, Math. Z. 56 (1952), 227-253.

[14] H. Heilbronn, On the class number in imaginary quadratic fields, Quart. J. Math. Oxford Ser. 25 (1934), 150-160.

[15] A. E. Ingham, The Distribution of Prime Numbers, Cambridge Tracts in Math. 30, Cambridge Univ. Press, Cambridge, 1990.

[16] H. Kestelman, Modern Theories of Integration, Dover, New York, 1960.

[17] N. Levinson and R. M. Redheffer, Complex Variables, Holden-Day, San Francisco, 1970.

[18] H. L. Montgomery and P. J. Weinberger, Notes on small class numbers, Acta Arith. 24 (1974), 529-542.

[19] J. Oesterlé, Nombres de classes des corps quadratiques imaginaires, Sém. Bourbaki, 1983-1984, exp. 631.

[20] C. L. Siegel, Über die Classenzahl quadratischer Zahlkörper, Acta Arith. 1 (1936), 83-86.

[21] —, Zum Beweise des Starkschen Satzes, Invent. Math. 5 (1968), 180-191.

[22] H. M. Stark, On complex quadratic number fields with class number equal to one, Trans. Amer. Math. Soc. 122 (1966), 112-119.

[23] - A complete determination of the complex quadratic fields of class number one, Michigan Math. J. 14 (1967), 1-27.

[24] - On the "gap" in a theorem of Heegner, J. Number Theory 1 (1969), 16-27.

[25] -, L-functions and character sums for quadratic forms (II), Acta Arith. 15 (1969), 307-317.

[26] - A transcendence theorem for class number problems, Ann. of Math. 94 (1971), 153-173.

[27] -, On a transcendence theorem for class number problems II, ibid. 96 (1972), 251259.

[28] -, On complex quadratic fields with class number two, Math. Comp. 29 (1975), 289-302.

[29] G. N. Watson, A Treatise on the Theory of Bessel Functions, 2nd ed., Cambridge Univ. Press, London, 1966.

[30] A. Weil, On some exponential sums, Proc. Nat. Acad. Sci. U.S.A. 34 (1948), 204220 .

[31] P. J. Weinberger, On small zeros of Dirichlet L-functions, Math. Comp. 29 (1975), 319-328.

Center for Computing Sciences

17100 Science Drive

Bowie, Maryland 20715

U.S.A.

E-mail: arno@super.org

robinson@super.org

wheeler@super.org

Received on 13.3 .1995

and in revised form on 15.12.1996 